\title{
Generalized coherent states and the diagonal representation for operators
}

\author{
N. Mukunda ${ }^{a)}$ \\ Centre for Theoretical Studies, Indian Institute of Science, Bangalore 560 012, India \\ and Jawaharlal Nehru Centre for Advanced Scientific Research, \\ Jakkur, Bangalore 560 064, India \\ Arvind ${ }^{\mathrm{b})}$ \\ Department of Physics, Guru Nanak Dev University, Amritsar 143005, India \\ S. Chaturvedic) \\ School of Physics, University of Hyderabad, Hyderabad 500 046, India \\ R. Simon ${ }^{\mathrm{d})}$ \\ The Institute of Mathematical Sciences, C. I. T. Campus, Chennai 600 113, India
}

\begin{abstract}
We consider the problem of existence of the diagonal representation for operators in the space of a family of generalized coherent states associated with a unitary irreducible representation of a (compact) Lie group. We show that necessary and sufficient conditions for the possibility of such a representation can be obtained by combining Clebsch-Gordan theory and the reciprocity theorem associated with induced unitary group representations. Applications to several examples involving $\mathrm{SU}(2), \mathrm{SU}(3)$, and the Heisenberg-Weyl group are presented, showing that there are simple examples of generalized coherent states which do not meet these conditions. Our results are relevant for phase-space description of quantum mechanics and quantum state reconstruction problems.
\end{abstract}

\section{INTRODUCTION}

There is a long history of attempts to express the basic structure of quantum mechanics, both kinematics and dynamics, in the $c$-number phase space language of classical mechanics. The first major step in this direction was taken by Wigner ${ }^{1}$ very early in the development of quantum mechanics, during a study of quantum corrections to classical statistical mechanics. This led to the definition of a real phase space distribution ${ }^{2}$ - now called the Wigner distribution - faithfully representing any pure or mixed state of a quantum system whose kinematics is governed by Heisenberg commutation relations for any number of Cartesian degrees of freedom. It was soon realized that this construction is dual to a rule proposed earlier by Weyl ${ }^{3}$ to map classical dynamical variables onto quantum mechanical operators in an unambiguous way, in the sense that the expectation value of any quantum operator in any quantum state can be rewritten in a completely $c$-number form on the corresponding classical phase space.

The general possibilities of expressing quantum mechanical operators in classical $c$-number forms were later examined by Dirac ${ }^{4}$ while developing the analogies between classical and quantum mechanics. The specific case of the Weyl-Wigner correspondence was carried further in important work by Groenewold and by Moyal. ${ }^{5}$

\footnotetext{
${ }^{a)}$ Electronic mail: nmukunda@cts.iisc.ernet.in

${ }^{b}$ Present address: Department of Physics, Carnegie Mellon University, Pittsburgh, PA 15213; Electronic mail: xarvind@andrew.cmu.edu, arvind@physics.iisc.ernet.in

${ }^{c}$ Electronic mail: scsp@uohyd.ernet.in

${ }^{\mathrm{d})}$ Electronic mail: simon@imsc.res.in
} 
Inspired by the needs of quantum optics, the general problem of setting up different classical variable-quantum operator correspondences has received enormous attention. ${ }^{6}$ It has thus been appreciated that the Weyl-Wigner choice is just one of many possibilities, two other important ones being (in the language of photon annihilation and creation operators) the normal ordering ${ }^{7}$ and the antinormal ordering ${ }^{8}$ choices.

While the harmonic oscillator coherent states, with their remarkable properties, have played a crucial role in all these developments, the idea of coherent states itself has been extended, in two slightly different ways, by Klauder ${ }^{9}$ and by Perelomov, ${ }^{10}$ to the notion of generalized coherent states. A very interesting case is the family of coherent states in the context of an unitary irreducible representation (UIR) of any Lie group $G$ on a Hilbert space $\mathcal{H}$. In particular, the generalized coherent states associated with the group $\mathrm{SU}(2)$ - the atomic coherent states ${ }^{11}$ - have received enormous attention. A satisfactory generalization of the concept of Wigner distribution has also been achieved for the irreducible representations of $\mathrm{SU}(2){ }^{12}$

For the purpose of the present work two different levels of completeness exhibited by the harmonic oscillator coherent states should be highlighted:

(i) Completeness at the state vector level: This refers to the fact that every state vector can be written as a linear combination of the coherent states. In terms of the (rank-one) operators corresponding to projections onto the coherent states, this property is expressed by saying that the identity operator can be expressed as a linear combination of these one-dimensional projection operators. This aspect is sometimes known as resolution of unity.

(ii) Completeness at the operator level: This refers to the remarkable fact that every operator can be realized as a linear combination of projections onto coherent states. This property is often known as the diagonal representation theorem. ${ }^{7}$ (The accompanying coefficient function of the linear expansion can in general be a very singular distribution. ${ }^{13}$ ) This diagonal representation of operators is dual to the normal ordering rule in the same sense as the Wigner and Weyl rules are dual to one another. We may note in passing that the diagonal representation has been central to many developments in quantum optics and laser physics. It also plays an important role in defining what is known as the $*$-product for operators. ${ }^{14}$

In view of the interest in generalized coherent states, it is important to know if these two levels of completeness apply to a given system of generalized coherent states or not. The (over) completeness property of the generalized coherent states at the state vector level usually (but not always) follows as a direct consequence of Schur's lemma: completeness at this level depends on whether the group representation under consideration possesses the square integrability property or not.

Completeness at the operator level-namely the question of whether the diagonal representation theorem applies to a given system of generalized coherent states - turns out to be considerably more subtle. In our opinion this question has hitherto not received the direct attention that it deserves (we should, however, invite the reader's attention to some very insightful remarks by Klauder and Skagerstam on this question ${ }^{15}$ ).

In an important recent work, Brif and Mann ${ }^{16}$ have carried out extensive harmonic analysis in the space of rank-one projections onto a system of generalized coherent states. However, they do not study the question of which systems of generalized coherent states admit a diagonal representation for operators and which do not. Indeed, they give no indication that there can be fairly simple systems of generalized coherent states for which the diagonal representation theorem does not apply-in the sense that there exist operators which cannot be written as linear combinations of rank-one projection operators over these coherent states.

The main aim of the present paper is to examine in detail the question of completeness at the operator level and to develop necessary and sufficient conditions which will ensure that all operators on the (relevant) Hilbert space can indeed be expanded in terms of projections onto the generalized coherent states. The non-triviality of this question renders some amount of careful and delicate analysis indispensable, but we would like to assure the reader that the effort is so rewarding as to give a complete answer to an issue of considerable physical importance.

For definiteness we deal with the situation where the group $G$ is compact, so that its chosen 
UIR acts on a Hilbert space $\mathcal{H}$ of finite dimension. (However in some of the examples we formally extend our methods to certain noncompact $G$.) The important tools in our analysis are the wellknown reciprocity theorem when one examines an induced representation of $G^{17}$ arising from some UIR of a subgroup $H \subset G$ and asks for the occurrence and multiplicity of various UIR's of $G$ itself; and the structure of the Clebsch-Gordan series and coefficients for direct products of UIR's of $G$, in a form adapted to $H$. We will show that while the necessary and sufficient conditions mentioned above are met in certain cases of SU(2) and the Heisenberg-Weyl (HW) group, there are quite simple examples in the cases of $\mathrm{SU}(2)$ and $\mathrm{SU}(3)$ where they are not satisfied. This will attest to the necessity and significance of the conditions that we develop.

The contents of this paper are organized as follows. In Sec. II we set up the basic notations and definitions of generalized coherent states within a UIR of a general compact Lie group $G$, the two associated stability groups and coset spaces, and carry out the harmonic analysis at the vector level. The two distinct kinds of relationships between the stability groups are also carefully defined. Section III discusses the detailed properties of the projection operators onto the generalized coherent states, and performs the corresponding harmonic analysis. Using these and other results pertaining to the Clebsch-Gordan problem, we are able to obtain explicit necessary and sufficient conditions for existence of the diagonal representation in any given situation. In Sec. IV we consider applications to both $\mathrm{SU}(2)$ and $\mathrm{SU}(3)$, taking three examples in each case. The aim is to show how to check our conditions in practical cases, and to exhibit some simple situations where the diagonal representation exists, and other equally simple ones where it does not. Section $\mathrm{V}$ analyzes the Heisenberg-Weyl group in a heuristic way, to display how our conditions work and lead to expected results. Section VI contains concluding remarks. Appendixes A and B gather material on general Clebsch-Gordan series and coefficients, unit tensor operators, induced representation theory and the reciprocity theorem, for the convenience of the reader and to set up notations.

\section{HARMONIC ANALYSIS ON COSET SPACES-THE VECTOR LEVEL}

Let $G$ be an $n$-dimensional compact Lie group. As described in Appendix A, we denote the various UIR's (upto equivalence) of $G$ by a symbol $J$; within a UIR we denote a complete set of orthonormal basis labels (magnetic quantum numbers) by $M$. Both $J$ and $M$ stand in general for sets of several independent indices. Certain specific choices of the latter will be indicated later.

Let the Hilbert space $\mathcal{H}^{\left(J_{0}\right)}$ carry the $N_{J_{0}}$-dimensional UIR $\mathcal{D}^{\left(J_{0}\right)}(\cdot)$ of $G$. Choose and keep fixed some fiducial unit vector $\psi_{0} \in \mathcal{H}^{\left(J_{0}\right)}$. The orbit of $\psi_{0}$ is the collection of vectorsgeneralized coherent states- $\psi(g) \in \mathcal{H}^{\left(J_{0}\right)}$ obtained by acting on $\psi_{0}$ with all $g \in G$ :

$$
\vartheta\left(\psi_{0}\right)=\left\{\psi(g)=\mathcal{D}^{\left(J_{0}\right)}(g) \psi_{0} \mid g \in G\right\} \subset \mathcal{H}^{\left(J_{0}\right)} .
$$

Similarly, if $\rho_{0}=\psi_{0} \psi_{0}^{\dagger}$ is the pure state density matrix corresponding to $\psi_{0}$, its orbit in the space of all density matrices is

$$
\vartheta\left(\rho_{0}\right)=\left\{\rho(g)=\mathcal{D}^{\left(J_{0}\right)}(g) \rho_{0} \quad \mathcal{D}^{\left(J_{0}\right)}(g)^{\dagger}=\psi(g) \psi(g)^{\dagger} \mid g \in G\right\} .
$$

Two important subgroups $H_{0}, H$ in $G$ are now defined

$$
\begin{gathered}
H_{0}=\left\{g \in G \mid \mathcal{D}^{\left(J_{0}\right)}(g) \psi_{0}=\psi_{0}\right\} \subset G, \\
H=\left\{g \in G \mid \mathcal{D}^{\left(J_{0}\right)}(g) \psi_{0}=(\text { phase }) \quad \psi_{0}\right\} \subset G .
\end{gathered}
$$

The dependences of $H_{0}, H$ on $\psi_{0}$ are left implicit. The subgroup $H_{0}$ is the stability group of $\psi_{0}$ in the strict sense, while $H$ is the stability group of $\psi_{0}$ upto phase factors. On the other hand, $H$ is the stability group of $\rho_{0}$ in the strict sense:

$$
H=\left\{g \in G \quad \mid \rho(g)=\rho_{0}\right\} \subset G .
$$


By standard arguments one has the identifications of the two orbits with corresponding coset spaces of $G$ :

$$
\begin{gathered}
\vartheta\left(\psi_{0}\right) \simeq G / H_{0}=\Sigma_{0}, \\
\vartheta\left(\rho_{0}\right) \simeq G / H=\Sigma .
\end{gathered}
$$

For definiteness we always take coset spaces to be made up of right cosets $g H_{0}, g H$ in the two cases.

It is evident that $H_{0}$ is an invariant subgroup of $H$, and we can distinguish two qualitatively different situations depending on the nature of the quotient $H / H_{0}$ :

$$
\begin{aligned}
& \text { case A: } \quad H / H_{0}=\text { trivial or discrete, } \\
& \text { case B: } \quad H / H_{0}=\mathrm{U}(1) .
\end{aligned}
$$

These two possibilities can be pictured as follows: There is an obvious and natural projection map $\pi: \vartheta\left(\psi_{0}\right) \rightarrow \vartheta\left(\rho_{0}\right)$ or $\pi: \Sigma_{0} \rightarrow \Sigma$. (Since $H_{0}$ is a subgroup of $H$, every $H_{0}$-coset lies within some $H$-coset.) With respect to this projection map, in case A for each $\rho \in \vartheta\left(\rho_{0}\right)$, there is just one or a discrete set of vectors $\psi \in \pi^{-1}(\rho) \subset \vartheta\left(\psi_{0}\right)$; while in case B $\pi^{-1}(\rho)$ consists of all vectors $\left\{e^{i \alpha} \psi\right\}$ for some fixed $\psi$ and $0 \leqslant \alpha<2 \pi$. Stated in yet another manner: in case A with the help of action by elements in $G$ the phase of $\psi_{0}$ [and so of any $\psi(g)$ ] can be altered in only a discrete set of ways or not at all; and in case B these phases can be altered in a continuous manner, so that each $\pi^{-1}(\rho)$ contains a "U(1)-worth of vectors."

We now wish to exploit the results of harmonic analysis arising from the natural UR's of $G$ acting on square integrable functions on the two coset spaces $\Sigma_{0}, \Sigma$ in order to extract the $G$ representation contents of $\psi(g), \rho(g)$, respectively. The key point is that while both $\psi(g)$ and $\rho(g)$ have already known dependences on $g$, since they are obtained from $\psi_{0}$ and $\rho_{0}$, respectively, by actions via the given UIR $\mathcal{D}^{\left(J_{0}\right)}$ of $G$ [and in particular $\psi(g)$ for different $g$ may not be orthogonal, $\rho(g)$ for different $g$ may not be trace orthogonal], they are linear quantities. Namely each of them belongs to a corresponding linear space. Therefore natural complete orthonormal sets of functions on $\Sigma_{0}, \Sigma$ can be profitably used to project out the irreducible Fourier components of $\psi(g), \rho(g)$, respectively, with well defined irreducible behaviors under $G$, and then to resynthesize them. In the remainder of this section we look at the case of $\psi(g)$, i.e., we consider the situation at the vector level. In the following section we take up the case of $\rho(g)$ at the operator level.

We have seen that the two distinct possibilities for the quotient $H / H_{0}$ are given by Eq. (2.6). For simplicity in case A we limit ourselves to $H=H_{0}$, i.e., we will hereafter consider just two possibilities:

$$
\begin{array}{ll}
\text { case (a): } & H=H_{0} ; \\
\text { case (b): } & H / H_{0}=\mathrm{U}(1) .
\end{array}
$$

In case (b) we have $H \simeq H_{0} \times \mathrm{U}(1)$ apart possibly for some global identification rules. The intermediate case of $H / H_{0}$ discrete nontrivial can be handled by straightforward modifications of the analysis to follow. In case (a) the coset spaces $\Sigma_{0}, \Sigma$ coincide; and the harmonic analysis to be now developed for functions on $\Sigma$ to study $\psi(g)$ can later be used to study $\rho(g)$. In case (b), since $H$ is larger than $H_{0}$ by exactly one $\mathrm{U}(1)$ angle, the coset space $\Sigma_{0}$ is also larger than $\Sigma$ by (locally) one angle variable in the range $(0,2 \pi)$. Whereas for $\psi(g)$ we can use the results of harmonic analysis arising from appropriate UR's of $G$ on $\Sigma_{0}$ or on $\Sigma$, for $\rho(g)$ we have to use the results on $\Sigma$ alone. At this point, focusing on $\psi(g)$ we divide the discussion into cases (a) and (b). 


\section{A. Harmonic analysis in case (a): $H=H_{0}$}

With respect to $H \subset G$ the significant information available about the properties of the generalized coherent state vectors $\psi(g) \in \vartheta\left(\psi_{0}\right)$ can be summarized as follows:

$$
\begin{gathered}
h \in H: \quad \mathcal{D}^{\left(J_{0}\right)}(h) \psi_{0}=\psi_{0}, \\
\psi(g)=\mathcal{D}^{\left(J_{0}\right)}(g) \psi_{0}, \\
\psi(g h)=\psi(g), \\
\mathcal{D}^{\left(J_{0}\right)}\left(g^{\prime}\right) \psi(g)=\psi\left(g^{\prime} g\right) .
\end{gathered}
$$

Let us denote a general point on $\Sigma$, a general $H$-coset, by $q=g H$. The identity coset $e H=H$ is the distinguished origin $q_{0} \in \Sigma$. A general $g^{\prime} \in G$ maps $q$ to $q^{\prime}=g^{\prime} q$. Also denote by $\ell(q) \in G$ a (local) choice of coset representatives $\Sigma \rightarrow G$ :

$$
q \in \Sigma \rightarrow \ell(q) \in G: \quad \ell(q) q_{0}=q .
$$

(In general, considering that $G$ is a principal fiber bundle over $\Sigma$ as base and $H$ as fiber and structure group, such coset representatives are definable only locally, and not in a globally smooth way; however these aspects involving domains of definition and overlap transition functions can be taken care of suitably.) Then the independent information contained in the vectors $\psi(g)$ can be reexpressed as follows:

$$
\begin{gathered}
\psi_{0}(q)=\psi(\ell(q)), \quad \psi_{0}\left(q_{0}\right)=\psi_{0}, \\
\mathcal{D}^{\left(J_{0}\right)}(g) \psi_{0}(q)=\psi(g \quad \ell(q))=\psi(\ell(g q))=\psi_{0}(g q) .
\end{gathered}
$$

Based on these relationships we set up a UR of $G$ on functions on $\Sigma$ in this manner. The Hilbert space of the UR is

$$
L^{2}(\Sigma, C)=\left\{\left.f(q) \in C\left|\int_{\Sigma} d \mu(q)\right| f(q)\right|^{2}<\infty\right\} .
$$

Here $d \mu(q)$ is the $G$-invariant integration volume element on $\Sigma, d \mu(g q)=d \mu(q)$; in the case of compact $G$ and $H$ we assume it is normalized to unit total volume for $\Sigma$. On these (scalar valued) functions $f(q)$ we define the action of $G$ by unitary operators $\mathcal{U}(g)$ :

$$
(\mathcal{U}(g) f)(q)=f\left(g^{-1} q\right) .
$$

It is now recognized that we have here the UR $\mathcal{D}^{(\text {ind,o) }}$ of $G$ induced from the identity or trivial one-dimensional UIR of $H$, as described in Appendix B, Eq. (B4). (The superscript 0 is a reminder that the induction is from the trivial representation of $H$.) As explained there, by the well-known reciprocity theorem this UR $\mathcal{D}^{(\text {ind,0) }}$ of $G$ contains a general UIR $\mathcal{D}^{(J)}$ of $G$ as many times as the latter contains the trivial one-dimensional UIR of $H$. To make this quite explicit, at this point we choose the magnetic quantum number $M$ within UIR's of $G$ to consist of a triple $M=\mu j \mathrm{~m}$ : here $\mu$ is a multiplicity label for UIR's of $H, j$ is a label for UIR's of $H$, and $m$ is a magnetic quantum number within the $j$ th UIR of $H$. (As with $J$ and $M$, here too $j$ and $m$ in general stand for sets of several quantum numbers each.) Then the general matrix element within the $J$ th UIR of $G$ appears, adapted to $H$, as

$$
\mathcal{D}_{M M^{\prime}}^{(J)}(g)=\mathcal{D}_{\mu j m, \mu^{\prime} j^{\prime} m^{\prime}}^{(J)}(g) .
$$

With this information we have the result that a complete orthonormal basis for the Hilbert space $L^{2}(\Sigma, C)$ is given by 


$$
\begin{gathered}
Y_{\mu j m}^{(J \lambda)}(q)=N_{J}^{1 / 2} \mathcal{D}_{\mu j m, \lambda 00}^{(J)}(\ell(q)), \\
Y_{\mu j m}^{(J \lambda)}\left(q_{0}\right)=N_{J}^{1 / 2} \delta_{\lambda \mu} \delta_{j 0} \delta_{m 0} .
\end{gathered}
$$

(Here again $j=m=0$ corresponds to the identity UIR of $H$.) We can say that there are as many independent spherical harmonics on $\Sigma$ of representation type $J$ as $\mathcal{D}^{(J)}$ contains $H$-scalar states, and $\lambda$ counts this multiplicity. The basic properties of these functions are

$$
\begin{gathered}
Y_{\mu j m}^{(J \lambda)}(g q)=\sum_{\mu^{\prime} j^{\prime} m^{\prime}} \mathcal{D}_{\mu j m, \mu^{\prime} j^{\prime} m^{\prime}}^{(J)}(g) Y_{\mu^{\prime} j^{\prime} m^{\prime}}^{(J \lambda)}(q), \\
\int_{\Sigma} d \mu(q) Y_{\mu^{\prime} j^{\prime} m^{\prime}}^{\left(J^{\prime} \lambda^{\prime}\right)}(q)^{*} Y_{\mu j m}^{(J \lambda)}(q)=\delta_{J^{\prime} J^{\prime} \delta_{\lambda^{\prime} \lambda}} \delta_{\mu^{\prime} \mu} \delta_{j^{\prime} j} \delta_{m^{\prime} m}, \\
\sum_{J \lambda \mu j m} Y_{\mu j m}^{(J \lambda)}(q) Y_{\mu j m}^{(J \lambda)}\left(q^{\prime}\right)^{*}=\delta\left(q^{\prime}, q\right) .
\end{gathered}
$$

In the last completeness relation we have the Dirac delta function on $\Sigma$ with respect to the volume element $d \mu(q)$.

Now we use the above tools to perform the harmonic analysis of $\psi_{0}(q)$. The results, as may be expected, will be simple, but the pattern for the later treatment of $\rho(g)$ will be set. Let us denote an orthonormal basis for $\mathcal{H}^{\left(J_{0}\right)}$, adapted to $H$, by $\Psi_{\mu j m}^{\left(J_{0}\right)}$,

$$
\begin{gathered}
\mathcal{D}^{\left(J_{0}\right)}(g) \Psi_{\mu j m}^{\left(J_{0}\right)}=\sum_{\mu^{\prime} j^{\prime} m^{\prime}} \mathcal{D}_{\mu^{\prime} j^{\prime} m^{\prime}, \mu j m}^{\left(J_{0}\right)}(g) \Psi_{\mu^{\prime} j^{\prime} m^{\prime}}^{\left(J_{0}\right)}, \\
\Psi_{\mu^{\prime} j^{\prime} m^{\prime}}^{\left(J_{0}\right) \dagger} \Psi_{\mu j m}^{\left(J_{0}\right)}=\delta_{\mu^{\prime} \mu} \delta_{j^{\prime} j} \delta_{m^{\prime} m} .
\end{gathered}
$$

Since $\psi_{0}$ is an $H$-invariant vector in $\mathcal{H}^{\left(J_{0}\right)}$, it follows that the UIR $\mathcal{D}^{\left(J_{0}\right)}$ of $G$ contains at least one $H$-scalar state. Let us for simplicity choose $\psi_{0}$ to be the one corresponding to the multiplicity label $\mu$ having the value unity

$$
\psi_{0}=\Psi_{100}^{\left(J_{0}\right)} .
$$

Then the generalized coherent states $\psi(g)$, and hence $\psi_{0}(q)$, can be written out in explicit detail,

$$
\begin{aligned}
& \psi(g)=\mathcal{D}^{\left(J_{0}\right)}(g) \psi_{0}=\sum_{\mu j m} \mathcal{D}_{\mu j m, 100}^{\left(J_{0}\right)}(g) \Psi_{\mu j m}^{\left(J_{0}\right)}, \\
& \psi_{0}(q)=\psi(\ell(q))=N_{J_{0}}^{-1 / 2} \sum_{\mu j m} Y_{\mu j m}^{\left(J_{0}, 1\right)}(q) \Psi_{\mu j m}^{\left(J_{0}\right)} .
\end{aligned}
$$

We see that the Fourier coefficients of $\psi_{0}(q)$ are very simple:

$$
\int_{\Sigma} d \mu(q) Y_{\mu j m}^{(J \lambda)}(q)^{*} \psi_{0}(q)=N_{J_{0}}^{-1 / 2} \delta_{J J_{0}} \delta_{\lambda, 1} \Psi_{\mu j m}^{\left(J_{0}\right)}
$$

This is as expected, and the expansion of $\psi_{0}(q)$ in the complete set $\left\{Y_{\mu j m}^{(J \lambda)}(q)\right\}$ gives back the second of Eq. (2.18). 


\section{B. Harmonic analysis in case (b): $H \simeq H_{0} \times U(1)$}

Now $H_{0}$ and $H$ are distinct. The results expressed in Eqs. (2.18) and (2.19) remain valid and adequate as far as the harmonic analysis of $\psi(g)$ or $\psi_{0}(q)$ is concerned; we must just imagine $H$ and $\Sigma$ replaced throughout by $H_{0}$ and $\Sigma_{0}$ in the case (a) analysis. However since the larger subgroup $H$ is now available, we outline the kind of induced UR of $G$ we would have to set up on functions on the smaller coset space $\Sigma=G / H$, suitable for the harmonic analysis of $\psi(g)$ if one so wished.

With respect to $H \simeq H_{0} \times \mathrm{U}(1) \subset G$, in contrast to the previous Eq. (2.8), we can now say the following about the family of generalized coherent states:

$$
\begin{gathered}
h \in H: \quad \mathcal{D}^{\left({ }_{0}\right)}(h) \psi_{0}=e^{i \varphi(h)} \psi_{0}, \\
\varphi\left(h^{-1}\right)=-\varphi(h), \\
\varphi(h)=0 \quad \text { for } \quad h \in H_{0}, \\
\psi(g h)=e^{i \varphi(h)} \psi(g), \\
\mathcal{D}^{\left(J_{0}\right)}\left(g^{\prime}\right) \psi(g)=\psi\left(g^{\prime} g\right) .
\end{gathered}
$$

(The last statement here is the same as before.) Now let us denote a general $H$-coset, a point of $\Sigma$, by $r=g H$. (Since $H_{0} \neq H$, the symbol $q$ has been used up to label points of $\Sigma_{0}$.) The identity coset $e H=H$ is the distinguished origin $r_{0} \in \Sigma$; and $g^{\prime} \in G$ maps $r$ to $r^{\prime}=g^{\prime} r$. In local coordinates, the point $q \in \Sigma_{0}$ (the larger coset space) is a pair, $q=(r, \alpha)$ where $r \in \Sigma$ and $\alpha$ $\in[0,2 \pi)$ is the $\mathrm{U}(1)$ angle. Now let $\ell(r) \in G$ be a choice of (local) coset representatives $\Sigma$ $\rightarrow G$ :

$$
r \in \Sigma \rightarrow \ell(r) \in G: \quad \ell(r) r_{0}=r .
$$

Then the information (2.20) about the generalized coherent states $\psi(g)$ gets expressed in this way:

$$
\begin{gathered}
\widetilde{\psi}_{0}(r)=\psi(\ell(r)), \quad \widetilde{\psi}_{0}\left(r_{0}\right)=\psi_{0}, \\
\mathcal{D}^{\left(J_{0}\right)}(g) \widetilde{\psi}_{0}(r)=\mathcal{D}^{\left(J_{0}\right)}(g \ell(r)) \psi_{0}=\mathcal{D}^{\left(J_{0}\right)}\left(\ell(g r) \ell(g r)^{-1} g \ell(r)\right) \psi_{0}=e^{i \varphi\left(\ell(g r)^{-1} g \ell(r)\right)} \widetilde{\psi}_{0}(g r) .
\end{gathered}
$$

The characteristic difference compared to Eq. (2.10), namely the presence of the nontrivial phase factor, is to be noted. This means that for analyzing $\psi(g)$ in this setting we must construct a UR of $G$ on square integrable functions over $\Sigma$ involving a nontrivial multiplier. The Hilbert space of this representation is [for simplicity we use the same symbol $f$ as in Eq. (2.11)]:

$$
L^{2}(\Sigma, C)=\left\{\left.f(r) \in C\left|\int_{\Sigma} d \nu(r)\right| f(r)\right|^{2}<\infty\right\},
$$

where $d \nu(r)=d \nu(g r)$ is the $G$-invariant normalized volume element on $\Sigma$. [Therefore locally $d \mu(q)=(1 / 2 \pi) d \nu(r) d \alpha$.] On such $f(r)$ we set up a UR $\tilde{\mathcal{U}}(g)$ of $G$ as follows:

$$
(\widetilde{\mathcal{U}}(g) f)(r)=e^{i \varphi\left(\ell(r)^{-1} g \ell\left(g^{-1} r\right)\right)} f\left(g^{-1} r\right) .
$$

This is recognized to be the UR of $G$ induced from the nontrivial one-dimensional UIR $e^{i \varphi(h)}$ of $H$, in which $H_{0}$ is represented trivially. One can now proceed with the harmonic analysis of $\psi(g)$ in which the subgroup $H$ plays the key role, by starting from an orthonormal basis for $\mathcal{H}^{\left(J_{0}\right)}$ adapted to $H$ rather than merely to $H_{0}$. However as we have already performed the harmonic 
analysis of $\psi(g)$ with respect to its strict stability subgroup $H_{0}$, we do not pursue case (b) for $\psi(g)$ any further; these additional details will become relevant in the next section, and will be spelt out there.

\section{HARMONIC ANALYSIS FOR THE PROJECTIONS}

When we turn to an analysis of the projection operators $\rho(g)=\psi(g) \psi(g)^{\dagger}$ we see that in both cases (a) and (b) the analysis must be based on the strict stability group $H$ of $\rho_{0}$, and therefore with the appropriate induced UR of $G$ on functions over $\Sigma$. (Thus uniformly the vector level analysis is better done using $H_{0}$, and the operator level analysis using $H$, whatever the relationship between $H_{0}$ and $H$ may be.) The results of the harmonic analysis are now not as simple as for $\psi(g)$ in Eqs. (2.18) and (2.19). We now treat the details as far as possible parallel to the discussions in the preceding section, first for case (a) and then for case (b).

\section{A. Projection operators in case (a)}

The basic facts about the family of projection operators $\rho(g)$ are, in the pattern of Eqs. (2.8) and (2.20),

$$
\begin{gathered}
h \in H: \quad \mathcal{D}^{\left(J_{0}\right)}(h) \rho_{0} \mathcal{D}^{\left(J_{0}\right)}(h)^{\dagger}=\rho_{0}, \\
\rho(g)=\mathcal{D}^{\left(J_{0}\right)}(g) \rho_{0} \mathcal{D}^{\left(J_{0}\right)}(g)^{\dagger}, \\
\rho(g h)=\rho(g), \\
\mathcal{D}^{\left(J_{0}\right)}\left(g^{\prime}\right) \rho(g) \mathcal{D}^{\left(J_{0}\right)}\left(g^{\prime}\right)^{\dagger}=\rho\left(g^{\prime} g\right) .
\end{gathered}
$$

Using the notations for the coset space $\Sigma=G / H$ already introduced in the preceding section under case (a), and the coset representatives $\ell(q)$ in Eq. (2.9), we can express the content of Eqs. (3.1) as follows:

$$
\begin{gathered}
\rho_{0}(q)=\rho(\ell(q)), \quad \rho_{0}\left(q_{0}\right)=\rho_{0}, \\
\mathcal{D}^{\left(J_{0}\right)}(g) \rho_{0}(q) \mathcal{D}^{\left(J_{0}\right)}(g)^{\dagger}=\rho(g \ell(q))=\rho(\ell(g q))=\rho_{0}(g q) .
\end{gathered}
$$

For the harmonic analysis of $\rho(g)$ or $\rho_{0}(q)$ we therefore set up on $L^{2}(\Sigma, C)$, by Eq. (2.12), the induced UR $\mathcal{D}^{\text {(ind, }, 0)}(g)=\mathcal{U}(g)$ of $G$ just as was done for $\psi(g)$ in case (a). The UIR contents of this UR are as described in the preceding section. A complete orthonormal basis is provided by Eqs. (2.14) with the properties (2.15); so the UIR $\mathcal{D}^{(J)}$ of $G$ is present as many times as it contains $H$-scalar states, and the index $\lambda$ counts this multiplicity.

We can now project out the Fourier coefficients $\rho_{\mu j m}^{J \lambda}$ of $\rho(g)$ as operators acting on $\mathcal{H}^{\left(J_{0}\right)}$ :

$$
\rho_{\mu j m}^{J \lambda}=\int_{\Sigma} d \mu(q) Y_{\mu j m}^{(J \lambda)}(q)^{*} \rho_{0}(q)
$$

On the one hand, combined use of Eqs. (2.15) and (3.2) and unitarity of $\mathcal{D}^{(J)}$ leads to the expected tensor operator behavior:

$$
\mathcal{D}^{\left(J_{0}\right)}(g) \rho_{\mu j m}^{J \lambda} \mathcal{D}^{\left(J_{0}\right)}(g)^{\dagger}=\sum_{\mu^{\prime} j^{\prime} m^{\prime}} \mathcal{D}_{\mu^{\prime} j^{\prime} m^{\prime}, \mu j m}^{(J)}(g) \rho_{\mu^{\prime} j^{\prime} m^{\prime}}^{J \lambda} .
$$

On the other hand, the completeness relation in Eq. (2.15) gives

$$
\rho_{0}(q)=\sum_{J \lambda \mu j m} Y_{\mu j m}^{(J \lambda)}(q) \rho_{\mu j m}^{J \lambda}
$$


while of course $\rho(g)$ for general $g$ is obtained by going to the $H$ coset of $g$ :

$$
g=\ell(q) h, \quad q \in \Sigma, \quad h \in H: \quad \rho(g)=\rho_{0}(q) .
$$

However all this by no means implies that all the operators $\rho_{\mu j m}^{J \lambda}$ are nonvanishing. What is clear is that the UIR's $J$ of $G$ that appear as tensor operators in the harmonic analysis of $\rho(g)$ (and their corresponding multiplicities) must be some subset of the spectrum of UIR's of $G$ that are known to be contained in the induced UR $\mathcal{D}^{(\text {ind, }, 0)} \equiv \mathcal{U}(\cdot)$, as dictated by the reciprocity theorem. Indeed one can see immediately that, when $G$ and $H$ are both compact and $G / H$ is nontrivial, $\mathcal{H}^{\left(J_{0}\right)}$ is finite dimensional whereas $\mathcal{D}^{(\text {ind,0) }}$ is infinite dimensional; therefore only a finite subset of the $\rho_{\mu j m}^{J \lambda}$ can be nonzero.

To pin down further the tensor operators $\rho_{\mu j m}^{J \lambda}$ we relate them directly to the fiducial vector $\psi_{0} \in \mathcal{H}^{\left(J_{0}\right)}$ and to the generalized coherent states $\psi(g)$. We have introduced in Eq. (2.16) the orthonormal basis $\Psi_{\mu j m}^{\left(J_{0}\right)}$ for $\mathcal{H}^{\left(J_{0}\right)}$ adapted to $H$, and in Eq. (2.17) we have identified $\psi_{0}$ to be $\Psi_{100}^{\left(J_{0}\right)}$. This has given the explicit expressions $(2.18)$ for $\psi(g)$ and $\psi_{0}(q)$. Combining these various results and also using Eq. (2.14) we see that the integrand on the right-hand side in Eq. (3.3) is

$$
\begin{aligned}
Y_{\mu j m}^{(J \lambda)}(q)^{*} \rho_{0}(q)= & N_{J}^{1 / 2} \sum_{\substack{\mu^{\prime} j^{\prime} m^{\prime} \\
\mu^{\prime \prime} j^{\prime \prime} m^{\prime \prime}}} \Psi_{\mu^{\prime} j^{\prime} m^{\prime}}^{\left(J_{0}\right)} \Psi_{\mu^{\prime \prime} j^{\prime \prime} m^{\prime \prime}}^{\left(J_{0}\right)^{\dagger}} \\
& \times \mathcal{D}_{\mu^{\prime} j^{\prime} m^{\prime}, 100}^{\left(J_{0}\right)}(\ell(q)) \mathcal{D}_{\mu^{\prime \prime} j^{\prime \prime} m^{\prime \prime}, 100}^{\left(J_{0}\right)}(\ell(q))^{*} \mathcal{D}_{\mu j m, \lambda 00}^{(J)}(\ell(q))^{*} .
\end{aligned}
$$

For the product of the two $\mathcal{D}^{*}$ matrix elements we have the Clebsch-Gordan decomposition given in Eq. (A7) involving the Clebsch-Gordan coefficients of $G$ adapted to $H$ :

$$
\begin{aligned}
& \mathcal{D}_{\mu^{\prime \prime} j^{\prime \prime} m^{\prime \prime}, 100}^{\left(J_{0}\right)}(\ell(q)) * \mathcal{D}_{\mu j m, \lambda 00}^{(J)}(\ell(q)) *
\end{aligned}
$$

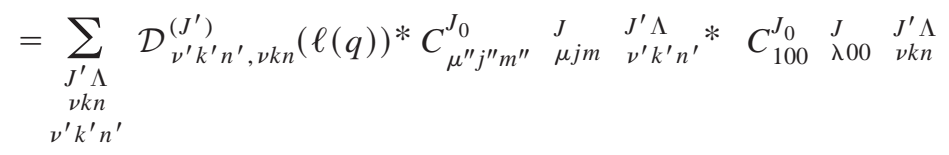

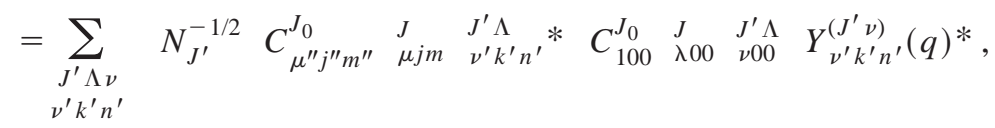

since the second Clebsch-Gordan coefficient shows that in the sums over $k$ and $n$ only $k=n$ $=0$ survives. Putting (3.8) into (3.3) and carrying out the integration we get the result

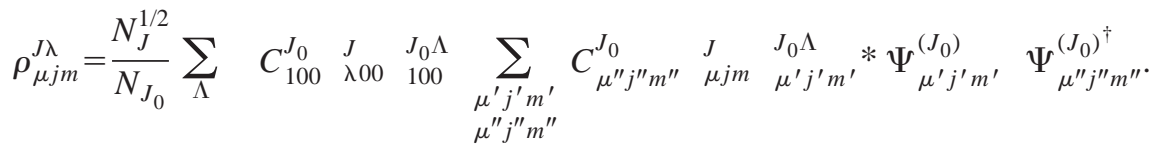

The sum over the outer products of the elements of the basis for $\mathcal{H}^{\left(J_{0}\right)}$ reproduces exactly the $\Lambda$ th unit tensor of rank $J$ on $\mathcal{H}^{\left(J_{0}\right)}$, as given in Eq. (A.12). Thus we have the final result

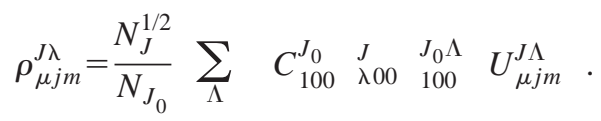

We immediately see that a necessary condition for $\rho_{\mu j m}^{J \lambda}$ to be nonzero is that the UIR $\mathcal{D}^{(J)}$ must occur in the direct product $\mathcal{D}^{J_{0}} \times \mathcal{D}^{\left(J_{0}\right)^{*}}$, which is of course reasonable.

It is also evident that a certain rectangular matrix for each $J$, made up of specific ClebschGordan coefficients, plays an important role here. We may write (3.10) as 


$$
\begin{gathered}
\rho_{\mu j m}^{J \lambda}=\sum_{\Lambda} \pi_{\lambda \Lambda}^{(J)} \quad U_{\mu j m}^{J \Lambda}, \\
\pi_{\lambda \Lambda}^{(J)}=\frac{N_{J}^{1 / 2}}{N_{J_{0}}} C_{100}^{J_{0}} \quad \begin{array}{lll}
{ }^{\prime} & & J_{0} \Lambda \\
100
\end{array} .
\end{gathered}
$$

The row index $\lambda$ gives the multiplicity of occurrence of $H$-scalar states within the UIR $\mathcal{D}^{(J)}$ of $G$, while the column index $\Lambda$ (which has no reference to $H$ ) gives the multiplicity of occurrence of $\mathcal{D}^{\left(J_{0}\right)}$ in the decomposition of the product $\mathcal{D}^{\left(J_{0}\right)} \times \mathcal{D}^{(J)}$. The necessary and sufficient conditions, in case (a), for being able to express every operator $A$ on $\mathcal{H}^{\left(J_{0}\right)}$ as an integral over the projections $\rho(g)$ or $\rho_{0}(q)$, namely, as

$$
A=\int_{\Sigma} d \mu(q) a(q) \rho_{0}(q)
$$

for some $c$-number function $a(q)$ depending linearly on $A$, are now clear. We know in advance that the set of unit tensor operators $U_{\mu j m}^{J \Lambda}$, with spectrum of $J \Lambda$ values completely and directly determined by $\mathcal{D}^{\left(J_{0}\right)}$ with no reference to the subgroup $H$, form a complete trace orthogonal set of operators on $\mathcal{H}^{\left(J_{0}\right)}$. Given the relations (3.11) for each $J$ expressing the Fourier coefficients of $\rho_{0}(q)$ in terms of these unit tensors, we must be able to invert these relations and express each $U_{\mu j m}^{J \Lambda}$ as a $\Lambda$-dependent linear combination over $\lambda$ of the $\rho_{\mu j m}^{J \lambda}$. Thus the necessary and sufficient conditions are as follows.

(i) Each UIR $\mathcal{D}^{(J)}$ of $G$ contained in the product UR $\mathcal{D}^{\left(J_{0}\right)} \times \mathcal{D}^{\left(J_{0}\right)^{*}}$ with some multiplicity must also occur in the UR $\mathcal{D}^{(\text {ind,0) }}$ of $G$ induced from the identity UIR of $H$, with the same or higher multiplicity.

(ii) For each such $\mathcal{D}^{(J)}$, the rectangular matrix $\pi^{(J)}$ in (3.11) must have at least as many rows as it has columns, and it must be of maximal rank, namely equal to the number of columns.

\section{B. Projection operators in case (b)}

The main complication now is that $\psi_{0}$ and $\rho_{0}$ have different strict stability groups. We therefore have to unavoidably introduce extra quantum numbers in the state labels to take account of the structure $H \simeq \mathrm{U}(1) \times H_{0}$. Further in carrying out harmonic analyses over $\Sigma=G / H$, we must use two different sets of complete orthonormal spherical harmonics, one appropriate for $\psi(g)$ and another (simpler) one for $\rho(g)$. The increase in index structure in $\mathcal{D}$-functions, $Y$-functions, and Clebsch-Gordan coefficients are all inevitable.

A general element $h \in H$ is a pair $h=\left(e^{i \alpha}, h_{0}\right)$ where $\alpha \in[0,2 \pi)$ and $h_{0} \in H_{0}$ (subject possibly to some global identification rules). The label $j$ for a general UIR of $H$ is also a pair $j$ $=\left(y, j_{0}\right)$ where $y \in \mathcal{Z}$ is the $\mathrm{U}(1)$ quantum number and $j_{0}$ labels a UIR of $H_{0}$ (again here $y$ and $j_{0}$ may be constrained in some way). Within the UIR $j_{0}$ of $H_{0}$ we have as before an internal magnetic quantum number $m$. Therefore in a basis adapted to $H$ the matrix elements in the UIR $\mathcal{D}^{(J)}$ of $G$ look like

$$
\mathcal{D}_{M M^{\prime}}^{(J)}(g)=\mathcal{D}_{\mu y j_{0} m, \mu^{\prime} y^{\prime} j_{0}^{\prime} m^{\prime}}^{(J)}(g),
$$

with the index $\mu$ counting the number of times the UIR $j \equiv\left(y, j_{0}\right)$ of $H$ is present, etc. Correspondingly we have an orthonormal basis $\Psi_{\mu y j_{0} m}^{\left(J_{0}\right)}$ for $\mathcal{H}^{\left(J_{0}\right)}$ with the transformation law

$$
\mathcal{D}^{\left(J_{0}\right)}(g) \Psi_{\mu y j_{0} m}^{\left(J_{0}\right)}=\sum_{\mu^{\prime} y^{\prime} j_{0}^{\prime} m^{\prime}} \mathcal{D}_{\mu^{\prime} y^{\prime} j_{0}^{\prime} m^{\prime}, \mu y j_{0} m}^{\left(J_{0}\right)}(g) \Psi_{\mu^{\prime} y^{\prime} j_{0}^{\prime} m^{\prime}}^{\left(J_{0}\right)}
$$


With no loss of generality we can assume that the fiducial vector $\psi_{0}$, invariant under $H_{0}$ but changing under the $\mathrm{U}(1)$ part of $H$, carries the $\mathrm{U}(1)$ quantum number $y=1$, and is the first such state in the case of multiplicity,

$$
\psi_{0}=\Psi_{1100}^{\left(J_{0}\right)}
$$

This replaces Eq. (2.17). For the generalized coherent state we have from Eq. (3.14) and (3.15), as replacement for Eq. (2.18):

$$
\psi(g)=\mathcal{D}^{\left(J_{0}\right)}(g) \Psi_{1100}^{\left(J_{0}\right)}=\sum_{\mu y j_{0} m} \mathcal{D}_{\mu y j_{0} m, 1100}^{\left(J_{0}\right)}(g) \Psi_{\mu y j_{0} m}^{\left(J_{0}\right)} .
$$

For points of the coset space $\Sigma$ and coset representatives we use the notations $r, \ell(r)$ already introduced in Sec. II under case (b). Now as was mentioned earlier, on $\Sigma$ we have to employ two different complete orthonormal sets of functions, one to handle $\psi_{0}(r)$ and the other to handle $\rho_{0}(r)$. This is because two different induced UR's of $G$ are involved-in the $\psi$ case it is the UR $\mathcal{D}^{\text {(ind,10) }}$ induced from the nontrivial one-dimensional UIR $j=(1,0)$ of $H$ as described in Eq. (2.24); in the $\rho$ case it is the UR $\mathcal{D}^{(\text {ind,00) }}$ induced from the trivial one-dimensional UIR $j$ $=(00)$ of $H$, analogous to Eq. (2.12). The two systems of complete orthonormal spherical harmonics on $\Sigma$ are

$$
\begin{array}{ll}
\mathcal{D}^{(\text {ind, } 10)}: & \widetilde{Y}_{\mu y j_{0} m}^{(J, \lambda)}(r)=N_{J}^{1 / 2} \mathcal{D}_{\mu y j_{0} m, \lambda 100}^{(J)}(\ell(r)), \\
\mathcal{D}^{(\text {ind, } 00)}: & Y_{\mu y j_{0} m}^{(J, \lambda)}(r)=N_{J}^{1 / 2} \mathcal{D}_{\mu y j_{0} m, \lambda 000}^{(J)}(\ell(r)) .
\end{array}
$$

We must appreciate that the spectrum of $(J, \lambda)$ values present in the two cases may be different, even though each set by itself is orthonormal and complete over $\Sigma$ with respect to the measure $d \nu(r)$. The transformation properties under $G$ action, orthonormality and completeness relations in each case are analogous to Eq. (2.15) and need not be repeated.

Equations (3.1) continue to hold, while we replace Eq. (3.2) and the second of Eq. (2.18) by

$$
\begin{gathered}
\rho_{0}(r)=\rho(\ell(r))=\widetilde{\psi}_{0}(r) \widetilde{\psi}_{0}(r)^{\dagger}, \\
\rho_{0}\left(r_{0}\right)=\rho_{0}, \\
\mathcal{D}^{\left(J_{0}\right)}(g) \rho_{0}(r) \mathcal{D}^{\left(J_{0}\right)}(g)^{\dagger}=\rho_{0}(g r), \\
\widetilde{\psi}_{0}(r)=\psi(\ell(r))=\sum_{\mu y j_{0} m} \mathcal{D}_{\mu y j_{0} m, 1100}^{\left(J_{0}\right)}(\ell(r)) \quad \Psi_{\mu y j_{0} m}^{\left(J_{0}\right)}=N_{J_{0}}^{-1 / 2} \sum_{\mu y j_{0} m} \widetilde{Y}_{\mu y j_{0} m}^{\left(J_{0}, 1\right)}(r) \Psi_{\mu y j_{0} m}^{\left(J_{0}\right)} .
\end{gathered}
$$

The pattern of calculations from here onwards is similar to case (a). We define the Fourier coefficients of the projection operators $\rho_{0}(r)$ with respect to the basis $(3.17 \mathrm{~b})$ as

$$
\begin{gathered}
\rho_{\mu y j_{0} m}^{J \lambda}=\int_{\Sigma} d \nu(r) \quad Y_{\mu y j_{0} m}^{(J, \lambda)}(r)^{*} \rho_{0}(r), \\
\rho_{0}(r)=\sum_{J \lambda \mu y j_{0} m} Y_{\mu y j_{0} m}^{(J, \lambda)}(r) \rho_{\mu y j_{0} m}^{J \lambda}, \\
\mathcal{D}^{\left(J_{0}\right)}(g) \quad \rho_{\mu y j_{0} m}^{J \lambda} \mathcal{D}^{\left(J_{0}\right)}(g)^{\dagger}=\sum_{\mu^{\prime} y^{\prime} j_{0}^{\prime} m^{\prime}} \mathcal{D}_{\mu^{\prime} y^{\prime} j_{0}^{\prime} m^{\prime}, \mu y j_{0} m}^{(J)}(g) \rho_{\mu^{\prime} y^{\prime} j_{0}^{\prime} m^{\prime}}^{J \lambda}
\end{gathered}
$$


We then use Eq. (3.18) to directly relate $\rho_{\mu y j_{0} m}^{J \lambda}$ to outer products of the basis vectors of $\mathcal{H}^{\left(J_{0}\right)}$, and then to the complete set of unit tensors on $\mathcal{H}^{\left(J_{0}\right)}$. Skipping the intermediate steps, the final result replacing Eq. (3.11) in case (a) is

$$
\begin{aligned}
& \rho_{\mu y j_{0} m}^{J \lambda}=\sum_{\Lambda} \pi_{\lambda \Lambda}^{(J)} U_{\mu y j_{0} m}^{J \Lambda}, \\
& \pi_{\lambda \Lambda}^{(J)}=\frac{N_{J}^{1 / 2}}{N_{J_{0}}} C_{1100}^{J_{0}} \quad \begin{array}{lll}
\lambda & J_{0} \Lambda \\
1100
\end{array},
\end{aligned}
$$

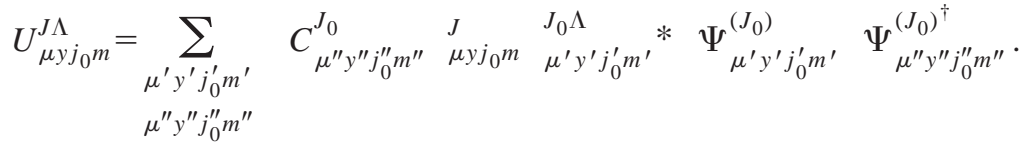

[For simplicity we have used the same symbols $\pi, U$ here as in case (a)]. The necessary and sufficient conditions to be able to express any operator $A$ on $\mathcal{H}^{\left(J_{0}\right)}$ as an integral over the projections $\rho(g)=\psi(g) \psi(g)^{\dagger}$ are now seen to read the same as in case (a), except that the family of rectangular matrices $\pi^{(J)}$ is specified in a different manner, and in condition (i) we have to read $\mathcal{D}^{(\text {ind,00) }}$ in place of $\mathcal{D}^{(\text {ind,0) }}$. For complete clarity, we state the two conditions explicitly: (i) Each UIR $\mathcal{D}^{(J)}$ of $G$ contained in the product UR $\mathcal{D}^{\left(J_{0}\right)} \times \mathcal{D}^{\left(J_{0}\right)^{*}}$ of $G$ with some multiplicity must also occur in the UR $\mathcal{D}^{(\text {ind,00) }}$ of $G$ induced from the identity UIR of $H \simeq U(1) \times H_{0}$, with the same or higher multiplicity. (ii) For each such $\mathcal{D}^{(J)}$, the rectangular matrix $\pi^{(J)}$ in (3.20) must have at least as many rows as it has columns, and it must be of maximal rank, namely equal to the number of columns.

In concluding this section we point out that we have made convenient choices of the vector $\psi_{0}$ in terms of a basis in $\mathcal{H}^{\left(J_{0}\right)}$, and this must be kept in mind since expressions for standard Clebsch-Gordan coefficients where available may differ from the ones needed in (3.11) and (3.20).

\section{APPLICATIONS TO SU(2) AND SU(3)}

As examples of the criteria developed in the last section for the existence of the diagonal coherent state representation for operators (in short, diagonal representation), we consider here some illustrative instances involving the simplest compact groups $\mathrm{SU}(2)$ and $\mathrm{SU}(3)$. Since the representation theory of these groups, their Clebsch-Gordan series and [at least for SU(2)] the Clebsch-Gordan coefficients are all well known, we describe very briefly the main features of each case considered. One point worth repeating is that the Clebsch-Gordan coefficients which appear in the criteria for existence of the diagonal representation through the matrices $\pi^{(J)}$ are generally noncanonical. We must bear in mind the use of bases for UIR's of $G$ adapted to the subgroup $H$ determined by $\psi_{0}$, and the identifications of $\psi_{0}$ in Eqs. (3.15) and (2.17). We look at three $\mathrm{SU}(2)$ cases and three $\mathrm{SU}(3)$ cases to illustrate the ideas.

\section{A. SU(2) Examples}

With $G=\mathrm{SU}(2)$, the Clebsch-Gordan series multiplicity label $\Lambda$ is absent, so we can set $\Lambda$ $=1$ everywhere. The UIR label $J$ has values $0,1 / 2,1, \ldots$ with $\mathcal{H}^{(J)}$ being of dimension $N_{J}=(2 J$ +1 ). We denote the generators by $T_{1}, T_{2}, T_{3}$. In discussing stability subgroups we pay attention only to the components continuously connected to the identity.

Example 1: Assume $J_{0} \geqslant 1$, and take $\psi_{0}$ to be a generic vector in $\mathcal{H}^{\left(J_{0}\right)}$, not an eigenvector of $\hat{n} \cdot T$ for any $\hat{n} \in S^{2}$. Independently of $\psi_{0}$, the spectrum of unit tensor operators on $\mathcal{H}^{\left(J_{0}\right)}$ is $J$ $=0,1,2, \ldots, 2 J_{0}$, once each. The stability groups are $H_{0}=H=\{e\}$, so we have case (a). The orbits $\vartheta\left(\psi_{0}\right), \vartheta\left(\rho_{0}\right)$ and the two coset spaces $\Sigma_{0}, \Sigma$ all coincide with $\mathrm{SU}(2)$ [or may be $\mathrm{S} 0(3)$ ] and are all three dimensional. Since $H$ is trivial, it has only the trivial one-dimensional UIR, so the induced representation $\mathcal{D}^{\text {(ind,0) }}$ of $\mathrm{SU}(2)$ is the regular representation $\mathcal{D}^{\text {(reg) }}$. The spectrum and 
multiplicity of UIR's present here is $J=0,1 / 2,1, \ldots, \infty, \mathcal{D}^{(J)}$ occurring $(2 J+1)$ times. Therefore condition (i) for case (a) is obeyed. Turning to condition (ii), any basis $\Psi_{\mu}^{(J)}, \mu=1,2, \ldots, 2 J+1$, in $\mathcal{H}^{(J)}$ is an $H$-adapted basis and $\mu$ is a multiplicity label. We take $\psi_{0}=\Psi_{1}^{\left(J_{0}^{\mu}\right)}$ in $\mathcal{H}^{(J)}$, assuming for definiteness that in each $\mathcal{H}^{(J)}$ we have a noncanonical basis (not eigenvectors of $T_{3}$ ). The matrices $\pi_{\lambda \Lambda}^{(J)}$ of Eq. (3.11) are column vectors with $(2 J+1)$ rows:

$$
\pi_{\lambda 1}^{(J)}=\frac{\sqrt{2 J+1}}{2 J_{0}+1} C_{1}^{J_{0}} \begin{array}{llll}
J & J_{0} & 1
\end{array}, \quad \lambda=1,2, \ldots, 2 J+1
$$

(We emphasize these are not the usual Clebsch-Gordan coefficients.) For each $J=0,1, \ldots, 2 J_{0}$ in the generic case we can expect this to be nonzero at least for one value of $\lambda$, as no particular symmetries or selection rules are operative. So condition (ii) also holds, and the diagonal representation exists.

Example 2: Assume $J_{0}$ is an integer $\geqslant 1$, and take $\psi_{0}$ to be the eigenvector of $T_{3}$ with eigenvalue $M_{0}=0$, i.e., in the canonical basis, $\psi_{0}=\Psi_{0}^{\left(J_{0}\right)}$. Again the spectrum of unit tensor operators on $\mathcal{H}^{\left(J_{0}\right)}$ is $J=0,1,2, \ldots, 2 J_{0}$, once each. The stability groups are $H_{0}=H=\mathrm{U}(1)$ generated by $T_{3}$, so we have case (a) again. Now we use the canonical basis $\Psi_{M}^{(J)}$ in every $\mathcal{H}^{(J)}$, as it is adapted to $H$; the multiplicity labels $\lambda, \mu$ are not needed, and can all be set equal to unity. The orbits $\vartheta\left(\psi_{0}\right), \vartheta\left(\rho_{0}\right)$ and the coset spaces $\Sigma_{0}, \Sigma$ all coincide with $\mathrm{SU}(2) / \mathrm{U}(1)=S^{2}$, and are all two dimensional. The induced UR $\mathcal{D}^{(\text {ind,0) }}$ of $\mathrm{SU}(2)$ is the helicity zero UR acting on functions on $S^{2}$, and this contains the UIR's $J=0,1,2, \ldots, \infty$, once each; thus condition (i) is obeyed. Turning to condition (ii), for each $J=0,1, \ldots, 2 J_{0}$ we have a single number $\pi_{11}^{(J)}$ to examine, and it is the canonical Clebsch-Gordan coefficient

$$
\pi_{11}^{(J)}=\frac{\sqrt{2 J+1}}{2 J_{0}+1} C_{0}^{J_{0}} \quad \begin{array}{lll}
J & J_{0} \\
0
\end{array}
$$

But it is known that this vanishes for $J=1,3, \ldots, 2 J_{0}-1$, hence condition (ii) is not obeyed, and the diagonal representation does not exist. This interesting situation was indeed noted by Klauder and Skagerstam a long time ago, ${ }^{15}$ for the case $J_{0}=1$.

Example 3: Take any $J_{0} \geqslant 1 / 2$, and $\psi_{0}$ to be an eigenvector of $T_{3}$ in $\mathcal{H}^{\left(J_{0}\right)}$ with eigenvalue $M_{0} \neq 0$. Thus in the canonical basis we have $\psi_{0}=\Psi_{M_{0}}^{\left(J_{0}\right)},\left|M_{0}\right|>0$. The spectrum of unit tensors on $\mathcal{H}^{\left(J_{0}\right)}$ is $J=0,1, \ldots, 2 J_{0}$; while the stability subgroups are $H_{0}=\{e\}, H=\mathrm{U}(1)$ generated by $T_{3}$, leading to case (b). In each $\mathcal{H}^{(J)}$ we can use the canonical basis, and the labels $\lambda$, $\mu$, are not needed. The orbit $\vartheta\left(\psi_{0}\right)$ and the coset space $\Sigma_{0}$ are three dimensional, while $\vartheta\left(\rho_{0}\right)$ and $\Sigma$ are $S^{2}$ as in example 2. The induced UR of SU(2) to be used for $\rho(g), \mathcal{D}^{(\text {ind,00) }}$ is again the helicity zero UR on functions on $S^{2}$, with the UIR spectrum $J=0,1,2, \ldots, \infty$, once each. So condition (i) of case (b) is obeyed. For $J=0,1, \ldots, 2 J_{0}$ we have to now examine the canonical Clebsch-Gordan coefficient [see Eq. (3.20)],

$$
\pi_{11}^{(J)}=\frac{\sqrt{2 J+1}}{2 J_{0}+1} C_{M_{0} O M_{0}}^{J_{0} \quad J_{0}},
$$

and as this is nonzero if $M_{0} \neq 0$, condition (ii) is obeyed and the diagonal representation exists.

In these three $\mathrm{SU}(2)$ examples, condition (i) was always obeyed; while in example 2 alone condition (ii) was violated. Now we look at three SU(3) examples, in one of which even condition (i) fails.

\section{B. SU(3) Examples}

With $G=\mathrm{SU}(3)$, the Clebsch-Gordan series multiplicity label $\Lambda$ is generally necessary. The UIR's are labeled by a pair of independent integers, $J=(p, q)$, with $\mathcal{H}^{(p, q)}$, having dimension $N_{(p, q)}=\frac{1}{2}(p+1)(q+1)(p+q+2)$. We will throughout use the canonical basis within each 
$\mathcal{H}^{(p, q)}$, labeled by the quantum numbers $I, I_{3}, Y$ of the isospin $\mathrm{SU}(2)$ and hypercharge $\mathrm{U}(1)$ subgroups of $\mathrm{SU}(3)$. We will be using two subgroups, namely $\mathrm{U}(1) \times \mathrm{U}(1)$ and $\mathrm{U}(2)$. The corresponding induced UIR's of SU(3), arising from the trivial UIR's of these subgroups, have the following contents as deduced from the reciprocity theorem:

$$
\begin{gathered}
\mathcal{D}_{\mathrm{U}(1) \times \mathrm{U}(1)}^{(\text {ind, } 0)}=\sum_{\substack{p, q=0 \\
p=q \bmod 3}}^{\infty} \oplus n_{p, q} \mathcal{D}^{(p, q)}, \\
n_{p, q}=(p+1, q+1)_{<} ; \\
\mathcal{D}_{U(2)}^{(\text {ind, }, 0)}=\sum_{p=0}^{\infty} \oplus \mathcal{D}^{(p, p)} .
\end{gathered}
$$

We take $J_{0}=(1,1)$ corresponding to the eight-dimensional octet or adjoint representation for the first two examples. The spectrum of unit tensor operators on $\mathcal{H}^{(1,1)}$ is known to be

$$
(p, q)=(0,0), \quad(1,1), \quad(1,1), \quad(3,0), \quad(0,3), \quad(2,2) .
$$

We look at two choices of $\psi_{0}$.

Example 4: Take $\psi_{0}=\Psi_{100}^{(1,1)}$. Then $H_{0}=H=\mathrm{U}(1) \times \mathrm{U}(1)$ and we have case (a). In the canonical basis $\Psi_{I}^{(p, q)}$ for UIR's of SU(3), $I_{3}$ and $Y$ determine a (one-dimensional) UIR of $H$, so $I$ is the multiplicity label $\lambda, \mu, \ldots$ of the general formalism. From Eq. (4.4a) we see that $\mathcal{D}_{\mathrm{U}(1) \times \mathrm{U}(1)}^{\text {(ind,0) }}$ contains $(0,0)$ once, $(1,1)$ twice, $(3,0)$ and $(0,3)$ once each, and $(2,2)$ three times. Condition (i) is then obeyed. Turning to condition (ii), for each of the $(p, q)$ pairs listed in Eq. (4.5) we must examine the matrix $\pi_{\lambda \Lambda}^{(J)}=\pi_{I \Lambda}^{(p, q)}$. These involve quite simple Clebsch-Gordan coefficients of $\mathrm{SU}(3)$, which in turn are Clebsch-Gordan coefficients of SU(2) times so-called isoscalar factors. We have the following results:

$$
\begin{aligned}
& (p, q)=(0,0): \quad \pi_{0,1}^{(0,0)}=1 / 8,
\end{aligned}
$$

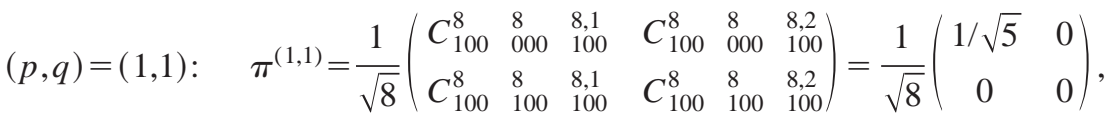

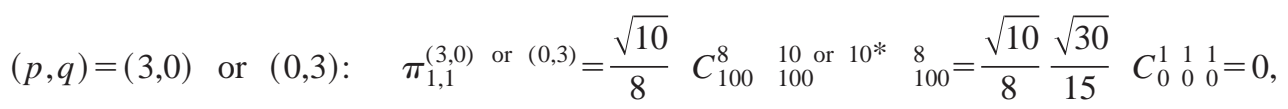

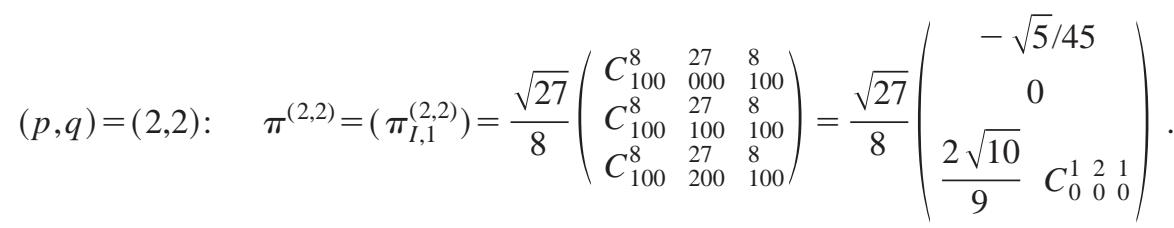

We see that condition (ii) fails for $(p, q)=(1,1),(3,0),(0,3)$, so the diagonal representation does not exist. It is noteworthy that in some cases we have the vanishing of the iso-scalar factor, and in other cases of the multiplying $\mathrm{SU}(2)$ coefficient.

Example 5: Take $\psi_{0}=\Psi_{000}^{(1,1)}$. Again, as $H_{0}=H=U(2)$, we have case (a). But now when we examine the contents of $\mathcal{D}_{U(2)}^{(\text {ind,0) }}$ in Eq. $(4.4 \mathrm{~b})$, we see that the UIR $(1,1)$ occurs just once, while $(3,0)$ and $(0,3)$ are both absent. This means that even condition (i) is not satisfied, and so the diagonal representation does not exist. 
Example 6: For the final SU(3) example, we choose the UIR $J_{0}=(3,0)$ with $N_{J_{0}}=10$. The fiducial state $\psi_{0}$ is taken to be the $\mathrm{SU}(2)$ scalar state with canonical quantum numbers $I I_{3} Y$ $=0,0,-2$ :

$$
\psi_{0}=\Psi_{00-2}^{(3,0)}
$$

[This is equivalent via an $\mathrm{SU}(3)$ transformation to choosing the highest weight state $\Psi_{3 / 2}^{(3,0)} 3 / 21$.] The corresponding stability groups are

$$
H_{0}=\mathrm{SU}(2), \quad H=\mathrm{U}(2),
$$

so this is an instance of case (b). The spectrum of unit tensors on $\mathcal{H}^{(3,0)}$ is determined by the Clebsch-Gordan series for the product $(3,0) \times(0,3)$ :

$$
(p, p)=(0,0), \quad(1,1), \quad(2,2), \quad(3,3),
$$

of dimensions $1,8,27$, and 64, respectively. Combining this with Eq. (4.4b) we see that in this example both kinds of multiplicity labels $\mu, \lambda, \ldots$ and $\Lambda$ are not needed, so all the relevant matrices $\left(\pi_{\lambda \Lambda}^{(J)}\right)$ reduce to single numbers. Comparing Eq. (4.9) with Eq. (4.4b) we see that condition (i) for existence of the diagonal representation is satisfied. Furthermore, since $I=I_{3}$ $=0$ for the fiducial vector $\psi_{0}$ in Eq. (4.7), the relevant Clebsch-Gordan coefficients reduce to just the isoscalar factors. The necessary coefficients are tabulated in closed form in Ref. 18 and we read off the values needed,

$$
\begin{aligned}
& \pi^{(3,3)}=\frac{8}{10} \quad C_{00-2}^{10} \quad \begin{array}{lll}
000 & 100-2
\end{array}=\frac{\sqrt{2}}{5 \sqrt{7}}, \\
& \pi^{(2,2)}=\frac{3 \sqrt{3}}{10} C_{00-2}^{10} \stackrel{27}{000} 00-2=\frac{3 \sqrt{3}}{10 \sqrt{7}}, \\
& \pi^{(1,1)}=\frac{2 \sqrt{2}}{10} C_{00-2}^{10} \stackrel{8}{800}{ }_{00-2}^{10}=\frac{1}{5}, \\
& \pi^{(0,0)}=\frac{1}{10} \quad C_{00-2}^{10} \quad \begin{array}{lll}
000 & 100-2
\end{array}=\frac{1}{10} .
\end{aligned}
$$

[For simplicity, as in Eq. (4.6), we have again indicated the relevant SU(3) UIR's by their dimensionalities, the 10 referring to $(3,0)]$. As these are all nonzero, condition (ii) is also satisfied, so we conclude that in this example the diagonal representation exists.

\section{THE HEISENBERG-WEYL GROUP}

The last application of our formalism is to the noncompact Heisenberg-Weyl (HW) group, denoted in this section by $G$. This will be somewhat heuristic as we shall often use Hilbert space vectors subject to delta-function normalization, induced representations whose reduction into UIR's involves continuous integrals, etc. The main aim is to show the relevance of the necessary and sufficient conditions of Sec. III for existence of the diagonal representation in this situation which underlies the very important case of ordinary coherent states. While the structure of $G$ (recalled below) is quite simple, its UIR's and the various Clebsch-Gordan series have quite delicate properties. We give a brief account of all these aspects.

Topologically $G$ has the structure of $\mathcal{R}^{3}$. Its Lie algebra $\underline{G}$ is spanned by three elements $t_{j}, j=1,2,3$, with the Lie bracket relations

$$
\left[t_{1}, t_{2}\right]=t_{3}, \quad\left[t_{1} \text { or } t_{2}, t_{3}\right]=0 \text {. }
$$


Finite group elements and the composition law and inverses are

$$
\begin{gathered}
\underline{\alpha}, \underline{\beta} \in \mathcal{R}^{3}: \quad g(\underline{\alpha})=\exp \left(\alpha_{2} t_{1}-\alpha_{1} t_{2}+\alpha_{3} t_{3}\right), \\
g(\underline{\alpha})^{-1}=g(-\underline{\alpha}), \\
g(\underline{\alpha}) g(\underline{\beta})=g\left(\alpha_{1}+\beta_{1}, \alpha_{2}+\beta_{2}, \alpha_{3}+\beta_{3}+\frac{1}{2}\left(\alpha_{1} \beta_{2}-\alpha_{2} \beta_{1}\right)\right) .
\end{gathered}
$$

In a UR or UIR we will write $-i T_{j}, T_{j}$ Hermitian, for $t_{j}$, so the generator commutation relations and unitary operators for finite group elements are

$$
\begin{gathered}
{\left[T_{1}, T_{2}\right]=i T_{3}, \quad\left[T_{1} \text { or } T_{2}, T_{3}\right]=0,} \\
g(\underline{\alpha}) \rightarrow \mathcal{D}(\underline{\alpha})=\exp \left(i\left(\alpha_{1} T_{2}-\alpha_{2} T_{1}-\alpha_{3} T_{3}\right)\right) .
\end{gathered}
$$

The adjoint action on the generators is

$$
\mathcal{D}(\underline{\alpha})\left(T_{1}, T_{2}, T_{3}\right) \mathcal{D}(\underline{\alpha})^{-1}=\left(T_{1}+\alpha_{1} T_{3}, T_{2}+\alpha_{2} T_{3}, T_{3}\right) .
$$

The UIR's of $G$ are of two types, depending on whether $T_{3}$ (which in any case is a scalar in a UIR) is zero or nonzero. If $T_{3}=0$, the UIR is one dimensional and is determined by choices of numerical values for $T_{1}, T_{2}$ :

$$
\mathcal{D}^{\left(q_{0}, p_{0}\right)}, \quad\left(q_{0}, p_{0}\right) \in \mathcal{R}^{2}: \quad T_{1}^{\left(q_{0}, p_{0}\right)}=q_{0}, \quad T_{2}^{\left(q_{0}, p_{0}\right)}=p_{0}, \quad T_{3}^{\left(q_{0}, p_{0}\right)}=0 .
$$

On the other hand, for $T_{3}=c \neq 0$, by the Stone-von Neumann theorem we have an infinitedimensional UIR on $L^{2}(\mathcal{R})$, acting on Schrödinger wave functions $\psi(q)$ of a real variable $q$ $\in \mathcal{R}$ as follows:

$$
\begin{gathered}
\mathcal{D}^{(c)}, \quad c \neq 0: \quad \mathcal{H}^{(c)}=L^{2}(\mathcal{R}), \\
T_{1}^{(c)}=\hat{q}=q, \quad T_{2}^{(c)}=\hat{p}=-i \quad c \frac{\partial}{\partial q}, \quad T_{3}^{(c)}=c .
\end{gathered}
$$

Thus there is an $\mathcal{R}^{2}$-worth collection of inequivalent one-dimensional UIR's $\mathcal{D}^{\left(q_{0}, p_{0}\right)}, \operatorname{dim} \mathcal{H}^{\left(q_{0}, p_{0}\right)}=1$; and an $\mathcal{R}-\{0\}$-worth collection of inequivalent infinite-dimensional UIR's $\mathcal{D}^{(c)}, \operatorname{dim} \mathcal{H}^{(c)}=\infty$. Every UIR is nonfaithful.

In the sequel, whenever there is no danger of confusion, we omit the UIR labels $\left(q_{0}, p_{0}\right)$ or $c$ on the generators $T_{j}$.

Turning to the Clebsch-Gordan problem, this is easily analyzed by examining the sums of the individual generators of any two UIR's. There are three cases to consider. The following two results are obvious:

$$
\begin{gathered}
\mathcal{D}^{\left(q_{0}, p_{0}\right)} \times \mathcal{D}^{\left(q_{0}^{\prime}, p_{0}^{\prime}\right)}=\mathcal{D}^{\left(q_{0}+q_{0}^{\prime}, p_{0}+p_{0}^{\prime}\right)}, \\
\mathcal{D}^{\left(q_{0}, p_{0}\right)} \times \mathcal{D}^{(c)}=\mathcal{D}^{(c)} .
\end{gathered}
$$

[In the latter case we may in fact appeal to Eq. (5.4)]. In the case of $\mathcal{D}^{(c)} \times \mathcal{D}^{\left(c^{\prime}\right)}$ we must distinguish between $c+c^{\prime}=0$ and $c+c^{\prime} \neq 0$. In either case the generators of the product, acting on $L^{2}\left(\mathcal{R}^{2}\right)$, are

$$
T_{1}=q+q^{\prime}, \quad T_{2}=-i \quad c \frac{\partial}{\partial q}-i \quad c^{\prime} \frac{\partial}{\partial q^{\prime}}, \quad T_{3}=c+c^{\prime} .
$$

For $c+c^{\prime} \neq 0$ we switch to the independent variables $Q=q+q^{\prime}, Q^{\prime}=c q^{\prime}-c^{\prime} q$, so 


$$
T_{1}=Q, \quad T_{2}=-i\left(c+c^{\prime}\right) \frac{\partial}{\partial Q}, \quad T_{3}=c+c^{\prime}
$$

We see that $Q^{\prime}$ is totally absent and commutes with all the $T_{j}$. In case $c+c^{\prime}=0$ we have

$$
T_{1}=q+q^{\prime}, \quad T_{2}=-i c\left(\frac{\partial}{\partial q}-\frac{\partial}{\partial q^{\prime}}\right), \quad T_{3}=0,
$$

(reminiscent of the EPR situation), and $T_{1}$ and $T_{2}$ form a complete commuting set. From all these results we see that

$$
\begin{gathered}
\mathcal{D}^{(c)} \times \mathcal{D}^{(-c)}=\iint_{\mathcal{R}^{2} \oplus d q_{0} d p_{0}} \mathcal{D}^{\left(q_{0}, p_{0}\right)}, \\
\mathcal{D}^{(c)} \times \mathcal{D}^{\left(c^{\prime}\right)}=\int_{\mathcal{R}} \oplus d Q^{\prime} \cdot \mathcal{D}^{\left(c+c^{\prime}\right)}, \quad c+c^{\prime} \neq 0 .
\end{gathered}
$$

In (5.11a) each one-dimensional UIR $\mathcal{D}^{\left(q_{0}, p_{0}\right)}$ appears once in a continuous fashion; while in (5.11b) the single infinite-dimensional UIR $\mathcal{D}^{\left(c+c^{\prime}\right)}$ appears infinitely often in a continuous sense, with $Q^{\prime}$ being a continuous multiplicity label. The full set of results for the Clebsch-Gordan problem is thus contained in Eqs. (5.7) and (5.11).

Now let us work within a particular UIR $\mathcal{D}^{(c)}$ acting on $\mathcal{H}^{(c)}$. From the results of the Clebsch-Gordan problem we see that the spectrum of irreducible unit tensors definable on $\mathcal{H}^{(c)}$ consists only of tensors belonging to the one-dimensional UIR's $\mathcal{D}^{\left(q_{0}, p_{0}\right)}$, once each in a continuous sense for every $\left(q_{0}, p_{0}\right) \in \mathcal{R}^{2}$. This is because $\mathcal{D}^{(c)} \times \mathcal{D}^{\left(c^{\prime}\right)}$ never contains $\mathcal{D}^{(c)}$, and $\mathcal{D}^{(c)}$ $\times \mathcal{D}^{\left(q_{0}, p_{0}\right)}$ is exactly $\mathcal{D}^{(c)}$. These unit tensors are the familiar $\mathrm{HW}$ displacement operators which are a subset of the unitary $\mathcal{D}^{(c)}(\underline{\alpha})$ themselves. The displacement operators are

$$
D^{(c)}\left(\alpha_{\perp}\right)=\mathcal{D}^{(c)}\left(\alpha_{\perp}, 0\right)=\exp \left(i \quad \alpha_{1} \hat{p}-i \quad \alpha_{2} \hat{q}\right),
$$

and for them the (finite form of the) adjoint action is

$$
\mathcal{D}^{(c)}(\underline{\beta}) D^{(c)}\left(\alpha_{\perp}\right) \mathcal{D}^{(c)}(\underline{\beta})^{-1}=e^{i c\left(\alpha_{1} \beta_{2}-\alpha_{2} \beta_{1}\right)} D^{(c)}\left(\underline{\alpha}_{\perp}\right)
$$

Therefore for each $\left(q_{0}, p_{0}\right) \in \mathcal{R}^{2}$ we define the (unitary) unit tensor operator

$$
U^{\left(q_{0}, p_{0}\right)}=D^{(c)}\left(\frac{q_{0}}{c}, \frac{p_{0}}{c}\right)
$$

(For simplicity we avoid the label $c$ on these operators.) Then from (5.13) we see that they have the correct transformation property, i.e., they belong to the one-dimensional UIR's $\mathcal{D}^{\left(q_{0}, p_{0}\right)}$ :

$$
\mathcal{D}^{(c)}(\underline{\alpha}) U^{\left(q_{0}, p_{0}\right)} \mathcal{D}^{(c)}(\underline{\alpha})^{-1}=e^{i\left(\alpha_{1} p_{0}-\alpha_{2} q_{0}\right)} \quad U^{\left(q_{0}, p_{0}\right)}=\mathcal{D}^{\left(q_{0}, p_{0}\right)}(\underline{\alpha}) \quad U^{\left(q_{0}, p_{0}\right)} .
$$

Moreover by familiar calculations, say in a basis of eigenvectors of $\hat{q}$, we can verify the trace orthonormality property in the delta function sense:

$$
\operatorname{Tr}\left(U^{\left(q_{0}^{\prime}, p_{0}^{\prime}\right)^{\dagger}} U^{\left(q_{0}, p_{0}\right)}\right)=2 \pi c \delta\left(q_{0}^{\prime}-q_{0}\right) \delta\left(p_{0}^{\prime}-p_{0}\right) .
$$

A general Hilbert-Schmidt operator $A$ on $\mathcal{H}^{(c)}$ can then be expanded as an integral over these unit tensors:

$$
A=\frac{\iint}{\mathcal{R}^{2}} d q_{0} \quad d p_{0} \quad a\left(q_{0}, p_{0}\right) \quad U^{\left(q_{0}, p_{0}\right)}
$$




$$
\begin{gathered}
a\left(q_{0}, p_{0}\right)=\frac{1}{2 \pi c} \operatorname{Tr}\left(U^{\left(q_{0}, p_{0}\right)^{\dagger}} A\right), \\
\operatorname{Tr}\left(A^{\dagger} A\right)=2 \pi c \int_{\mathcal{R}^{2}} d q_{0} d p_{0}\left|a\left(q_{0}, p_{0}\right)\right|^{2} .
\end{gathered}
$$

This is the Weyl representation for operators, and Eqs. (5.15)-(5.17) are the analogs in the present case for Eqs. (A13)-(A15) of the compact group case. All these results are available in advance of the choice of a fiducial vector, construction of its generalized coherent states, etc.

Now choose a fiducial unit vector $\psi_{0} \in \mathcal{H}^{(c)}$. From elementary quantum mechanics it is known that every real linear combination of $\hat{q}$ and $\hat{p}$ has a continuous spectrum and hence no normalizable eigenvectors. Therefore the stability group $H_{0}$ of $\psi_{0}$ is trivial. On the other hand, $H$ is $\mathcal{R}$ [but effectively just $\mathrm{U}(1)$ ] with generator $T_{3}$,

$$
\psi_{0} \in \mathcal{H}^{(c)}, \quad\left\|\psi_{0}\right\|=1: \quad H_{0}=\{e\}, \quad H=\left\{e^{-i \alpha_{3} c}, \quad \alpha_{3} \in \mathcal{R}\right\} .
$$

Thus for any $\psi_{0}$ we have case (b), and we have to examine the UIR content of the UR $\mathcal{D}^{\text {(ind,00) }}$ of $G$ induced from the trivial one-dimensional UIR of $H$ (namely, $T_{3}=0$ ). To apply the reciprocity theorem, we have to ask how often each UIR of $G$ contains the trivial UIR of $H$. Clearly each $\mathcal{D}^{\left(q_{0}, p_{0}\right)}$ contains it once, while each $\mathcal{D}^{(c)}$ does not contain it at all. In other words,

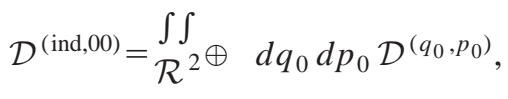

which matches exactly with the spectrum and multiplicity of irreducible tensor operators $U^{\left(q_{0}, p_{0}\right)}$ definable on $\mathcal{H}^{(c)}$, so condition (i) is satisfied. As for condition (ii), since $\mathcal{D}^{(c)} \times \mathcal{D}^{\left(q_{0}, p_{0}\right)}$ $=\mathcal{D}^{(c)}$, the quantity $\pi^{\left(q_{0}, p_{0}\right)}$ of Eq. (3.20) is just one number (disregarding the $N_{J_{0}}$ in the denominator), and the question is whether it is always nonvanishing - we examine this more directly as follows.

The generalized coherent states and projection operators arising from $\psi_{0}$ are

$$
\begin{gathered}
\psi(\underline{\alpha})=\mathcal{D}^{(c)}(\underline{\alpha}) \psi_{0}=e^{-i c \alpha_{3}} D^{(c)}\left(\alpha_{\perp}\right) \psi_{0}, \\
\rho\left(\alpha_{\perp}\right)=\psi(\underline{\alpha}) \psi(\underline{\alpha})^{\dagger}=D^{(c)}\left(\alpha_{\perp}\right) \rho_{0} D^{(c)}\left(\alpha_{\perp}\right)^{\dagger}, \\
\rho_{0}=\psi_{0} \quad \psi_{0}^{\dagger} .
\end{gathered}
$$

Under adjoint action we have

$$
\mathcal{D}^{(c)}(\underline{\beta}) \rho\left(\alpha_{\perp}\right) \mathcal{D}^{(c)}(\underline{\beta})^{-1}=\rho\left(\alpha_{\perp}+\beta_{\perp}\right),
$$

and $\beta_{3}$ is absent on the right. If we denote the Fourier transform of $\rho\left(\alpha_{\perp}\right)$ by

$$
\widetilde{\rho}\left(q_{0}, p_{0}\right)=\frac{1}{2 \pi} \iint_{\mathcal{R}}^{2} d^{2} \alpha e^{-i\left(\alpha_{1} p_{0}-\alpha_{2} q_{0}\right)} \rho\left(\alpha_{\perp}\right),
$$

then (5.21) becomes

$$
\mathcal{D}^{(c)}(\underline{\beta}) \quad \widetilde{\rho}\left(q_{0}, p_{0}\right) \mathcal{D}^{(c)}(\underline{\beta})^{-1}=e^{i\left(\beta_{1} p_{0}-\beta_{2} q_{0}\right)} \quad \widetilde{\rho}\left(q_{0}, p_{0}\right)=\mathcal{D}^{\left(q_{0}, p_{0}\right)}(\underline{\beta}) \quad \widetilde{\rho}\left(q_{0}, p_{0}\right) .
$$

Thus each $\widetilde{\rho}\left(q_{0}, p_{0}\right)$ is a tensor operator of type $\mathcal{D}^{\left(q_{0}, p_{0}\right)}$, consisting of just one component, so it must be a scalar multiple of the unit tensor $U^{\left(q_{0}, p_{0}\right)}$. This factor is easily computed by a trace calculation since by (5.17) the unit tensors are a complete orthonormal (in the continuous sense) set. An elementary calculation shows that 


$$
\operatorname{Tr}\left(U^{\left(q_{0}^{\prime}, p_{0}^{\prime}\right)^{\dagger}} \widetilde{\rho}\left(q_{0}, p_{0}\right)\right)=2 \pi\left(\psi_{0}, U^{\left(q_{0}, p_{0}\right)} \psi_{0}\right) * \delta\left(q_{0}-q_{0}^{\prime}\right) \delta\left(p_{0}-p_{0}^{\prime}\right),
$$

which gives the result

$$
\widetilde{\rho}\left(q_{0}, p_{0}\right)=\frac{1}{c}\left(\psi_{0}, U^{\left(q_{0}, p_{0}\right)} \psi_{0}\right) * U^{\left(q_{0}, p_{0}\right)}
$$

The necessary and sufficient condition for the existence of the diagonal representation in terms of the projections $\rho\left(\alpha_{\perp}\right)$ of Eq. (5.20) is now clear: the fiducial vector $\psi_{0}$ must be chosen so that for all $\left(q_{0}, p_{0}\right) \in \mathcal{R}^{2}$,

$$
\begin{aligned}
& \left(\psi_{0}, U^{\left(q_{0}, p_{0}\right)} \psi_{0}\right)=\left(\psi_{0}, D^{(c)}\left(\frac{q_{0}}{c}, \frac{p_{0}}{c}\right) \psi_{0}\right) \neq 0, \\
& \left(\psi_{0}, D^{(c)}\left(q_{0}, p_{0}\right) \psi_{0}\right)=\left(\psi_{0}, e^{i\left(q_{0} \hat{p}-p_{0} \hat{q}\right)} \psi_{0}\right) \neq 0 .
\end{aligned}
$$

Assuming this condition is satisfied, we can start from the Weyl representation (5.17) for any (Hilbert-Schmidt) operator $A$ and obtain from it a diagonal coherent state representation:

$$
\begin{aligned}
& A=\iint_{\mathcal{R}^{2}} d q_{0} d p_{0} a\left(q_{0}, p_{0}\right) \quad U^{\left(q_{0}, p_{0}\right)}=\iint_{\mathcal{R}^{2}} d q_{0} d p_{0} a\left(q_{0}, p_{0}\right) \quad c\left(\psi_{0}, U^{\left(q_{0}, p_{0}\right)^{\dagger}} \psi_{0}\right)^{-1} \quad \widetilde{\rho}\left(q_{0}, p_{0}\right) \\
& =\int_{\mathcal{R}^{2}} d^{2} \alpha \phi\left(\alpha_{\perp}\right) \rho\left(\alpha_{\perp}\right), \\
& \phi\left(\alpha_{\perp}\right)=\frac{c}{2 \pi} \iint_{\mathcal{R}^{2}} d q_{0} d p_{0} e^{i\left(\alpha_{2} q_{0}-\alpha_{1} p_{0}\right)} \quad a\left(q_{0}, p_{0}\right) /\left(\psi_{0}, U^{\left(q_{0}, p_{0}\right)} \psi_{0}\right)^{*} .
\end{aligned}
$$

From Eq. (5.17) we know that for Hilbert-Schmidt $A$, the function $a\left(q_{0}, p_{0}\right)$ is square integrable over $\mathcal{R}^{2}$; in relation to this, the nature of the weight function $\phi\left(\alpha_{\perp}\right)$ in the diagonal representation is determined by the factor $\left(\psi_{0}, U^{\left(q_{0}, p_{0}\right)} \psi_{0}\right) *$ in the denominator.

As an application we consider the case of the usual coherent states obtained when the fiducial vector $\psi_{0}$ is the Fock vacuum or the harmonic oscillator ground state. (Further, for simplicity we now set $c=1$.) The wave function is

$$
\psi_{0}(q)=\pi^{-1 / 4} e^{-q^{2} / 2},
$$

and a simple calculation gives the displacement operator expectation value needed in Eqs. (5.26) and (5.27),

$$
\left(\psi_{0}, U^{\left(q_{0}, p_{0}\right)} \psi_{0}\right)=\left(\psi_{0}, e^{i\left(q_{0} \hat{p}-p_{0} \hat{q}\right)} \psi_{0}\right)=e^{-\frac{1}{4}\left(q_{0}^{2}+p_{0}^{2}\right)} .
$$

This is indeed everywhere nonzero over $\mathcal{R}^{2}$, so the condition (5.26) for existence of the diagonal representation is, as expected, obeyed. The decaying exponential factor here means that the tensor operators $\widetilde{\rho}^{\left(q_{0}, p_{0}\right)}$ provided by the projection operators $\rho\left(\alpha_{\perp}\right)$ differ from the normalized unit tensors $U^{\left(q_{0}, p_{0}\right)}$ by similarly decaying factors:

$$
\widetilde{\rho}\left(q_{0}, p_{0}\right)=e^{-\frac{1}{4}\left(q_{0}^{2}+p_{0}^{2}\right)} U^{\left(q_{0}, p_{0}\right)} .
$$

It is to compensate for this diminishing norm of $\widetilde{\rho}\left(q_{0}, p_{0}\right)$ as one goes towards infinity in the $\left(q_{0}, p_{0}\right)$ phase plane that one finds that the weight function $\phi\left(\alpha_{\perp}\right)$, Eq. (5.27), has in general the character of a very singular distribution: the Fourier transform of $\phi\left(\alpha_{\perp}\right)$ is (essentially) the square integrable amplitude $a\left(q_{0}, p_{0}\right)$ times the exploding Gaussian $e^{\frac{1}{4}\left(q_{0}^{2}+q_{0}^{2}\right)}$. 
Another interesting choice of fiducial state for diagonal representation has been considered by Haake and Wilkens, ${ }^{19}$ namely the squeezed vacuum. The family of generalized coherent states in this case consists of Gaussian pure states squeezed by a fixed amount in a fixed direction in phase space, the center $\left(q_{0}, p_{0}\right)$ of the Gaussian being allowed to be located at an arbitrary point in phase space. It is easy to see that in this case $\left\langle\psi_{0}\left|D\left(q_{0}, p_{0}\right)\right| \psi_{0}\right\rangle$ is nonvanishing, and the diagonal representation once again exists:

$$
\begin{aligned}
\left|\psi_{0}\right\rangle & =S(\eta)|0\rangle, \quad S(\eta)=\exp \left(\frac{\eta}{2} \hat{a}^{\dagger} 2-\frac{\eta^{*}}{2} \hat{a}^{2}\right), \\
\left\langle\psi_{0}\left|D\left(q_{0}, p_{0}\right)\right| \psi_{0}\right\rangle & =\left\langle 0\left|S(\eta)^{-1} D\left(q_{0}, p_{0}\right) S(\eta)\right| 0\right\rangle \\
& =\left\langle 0\left|D\left(e^{\eta} q_{0}, e^{-\eta} p_{0}\right)\right| 0\right\rangle=\exp \left(-\frac{1}{4}\left(e^{2 \eta} q_{0}^{2}+e^{-2 \eta} p_{0}^{2}\right)\right) .
\end{aligned}
$$

Returning to the general result (5.27) whenever $\psi_{0}$ is an acceptable fiducial vector, we can appeal to the fact that the Stone-von Neumann UIR of the HW group is square integrable and conclude that $\left(\psi_{0}, U^{\left(q_{0}, p_{0}\right)} \psi_{0}\right)$ is a square integrable function of $\left(q_{0}, p_{0}\right)$. Thus this amplitude must approach zero as we move far away from the origin in $\mathcal{R}^{2}$. This has the consequence that, whatever the choice of $\psi_{0}$ [provided (5.26) holds], the weight function $\phi\left(\alpha_{\perp}\right)$ is in general a distribution, since in its Fourier representation (5.27) the square integrable amplitude $a\left(q_{0}, p_{0}\right)$ is divided by another square integrable amplitude.

We now make a series of statements which help in conveying the content of the condition (5.26) and in forming some (admittedly incomplete) idea of the set of fiducial vectors $\psi_{0}$ whose generalized coherent states are rich enough to allow for the diagonal representation:

(i) If $\psi_{0}(q)$ is any Gaussian wave function, then $\left(\psi_{0}, D^{(c)}\left(q_{0}, p_{0}\right) \psi_{0}\right)$ is clearly a complex Gaussian in $\left(q_{0}, p_{0}\right)$, so condition (5.26) is satisfied.

(ii) If $\psi_{0}$ does/does not obey condition (5.26), then the transform of $\psi_{0}$ by the unitary operator representing any element of the metaplectic group $M p(2)$ also does/does not obey condition (5.26). This is because under conjugation by such a unitary operator, $D^{(c)}\left(q_{0}, p_{0}\right)$ just becomes $D^{(c)}\left(q_{0}^{\prime}, p_{0}^{\prime}\right)$ for $\left(q_{0}^{\prime}, p_{0}^{\prime}\right)$ some linear combinations of $\left(q_{0}, p_{0}\right)$.

(iii) If either $\psi_{0}(q)$ or its Fourier transform $\widetilde{\psi}_{0}(p)$ is a function of compact support, then condition (5.26) is definitely not obeyed, so the diagonal representation will not exist. This is because for such $\psi_{0}$, the quantity $\left(\psi_{0}, D^{(c)}\left(q_{0}, p_{0}\right) \psi_{0}\right)$ vanishes outside a finite strip parallel to the $p_{0}$ or to the $q_{0}$ axis. We can also see that as Fourier transforms of functions of compact support are entire functions of a certain class, wave functions $\psi_{0}(q)$ of this class violate condition (5.26) quite strongly_indeed their Fourier transforms $\widetilde{\psi}_{0}(p)$ are of compact support.

In a purely qualitative manner we can appreciate now that Gaussian $\psi_{0}(q)$ and compactsupported $\psi_{0}(q)$ [or $\left.\widetilde{\psi}_{0}(p)\right]$ are in some ways diametrically opposite from the point of view of condition (5.26). To conclude this section we consider a set of fiducial vector choices where condition (5.26) is violated, though only on a set of measure zero in the $q_{0}-p_{0}$ plane. This will then mean that in these cases for Hilbert-Schmidt operators $A$ we do not have available the diagonal representation.

Consider the choice $|n\rangle$ for the fiducial vector $\psi_{0}$, this being the $n$th excited state of the harmonic oscillator, for $n \geqslant 1$. The resulting generalized coherent states are the displaced Fock states. ${ }^{20}$ It is known that the matrix element (or better expectation value) needed in condition (5.26) is essentially a Laguerre polynomial, thus

$$
\begin{gathered}
\left|\psi_{0}\right\rangle=|n\rangle: \\
\left\langle\psi_{0}\left|D^{(c)}\left(q_{0}, p_{0}\right)\right| \psi_{0}\right\rangle=e^{-\frac{1}{4}\left(q_{0}^{2}+p_{0}^{2}\right)} L_{n}\left(\frac{q_{0}^{2}+p_{0}^{2}}{2}\right) .
\end{gathered}
$$


Now, as is well known, the polynomial $L_{n}(x)$ has exactly $n$ distinct real zeroes in the semi-infinite interval $0<x<\infty$, hence the condition (5.26) is satisfied except on a discrete infinite sequence of circles in the $q_{0}-p_{0}$ plane. However, these singularities which are in the finite part of the $\left(q_{0}, p_{0}\right)$ plane are not integrable. Therefore we do not have the possibility of the diagonal representation for the above choices of $\psi_{0}$.

Recalling condition (5.26) for the existence of the diagonal representation, and the various examples discussed above, we are led in the Heisenberg-Weyl case to the conjecture that condition (5.26) is obeyed if and only if the fiducial state $\psi_{0}$ has Gaussian Schrödinger wave function. This will then mean that apart from the traditional diagonal representation and the Haake-Wilkens diagonal representation there are no other ones for the Heisenberg-Weyl group.

\section{CONCLUDING REMARKS}

We have developed necessary and sufficient conditions for a set of generalized coherent states, arising from a UIR of a compact Lie group to possess the property that a diagonal representation in terms of projections onto these states can be set up for any operator on the Hilbert space of the UIR. This has required combining several structures and properties-harmonic analysis on coset spaces, the theory of induced representations, the associated reciprocity theorem, and the Clebsch-Gordan problem and coefficients for the UIR's of the group under consideration. Each of these plays a crucial role in arriving at the complete set of conditions. The explicit examples involving $\mathrm{SU}(2), \mathrm{SU}(3)$ and even the Heisenberg-Weyl group show how our conditions operate in practice, and how we cannot do without any of the ingredients mentioned above. In particular it is important to appreciate that the examples where the diagonal representation fails to exist are not particularly exotic or contrived; and we can often see in advance those cases where it is bound to be absent.

The comprehensive work of Brif and Mann ${ }^{16}$ attempts also to exploit the methods of harmonic analysis on coset spaces to tackle the general closely related problems of Wigner distributions and state reconstruction problems. However, in the absence of detailed knowledge of the irreducible representation contents of various induced representations of $G$, it is easy to miss the fact that there are quite stringent conditions to be met before a diagonal representation can exist. The particular qualitative points to be made in connection with our approach are: for a given UIR of $G$, the complete set of irreducible unit tensor operators on the Hilbert space is immediately fixed, prior to construction of any set of generalized coherent states. As one then considers various choices of the fiducial vector $\psi_{0}$, one can see that for larger stability groups $H_{0}$ and $H$, the corresponding coset spaces $\Sigma_{0}$ and $\Sigma$ are smaller, with the consequence that the set of projection operators onto the generalized coherent states also becomes smaller, and so the diagonal representation is less likely to exist.

Finally we may mention that the issue of reproducing various marginal probability distributions out of a Wigner-type distribution description of density operators has played no role in our considerations. This, the application of our methods to phase space description of quantum systems, quantum state reconstruction (tomography), and other aspects of Wigner distributions for quantum mechanics on Lie groups will be systematically studied elsewhere.

\section{APPENDIX A: NOTATIONS FOR GROUP REPRESENTATIONS, CLEBSCH-GORDAN COEFFICIENTS, AND UNIT TENSORS}

In this Appendix we collect some items of notation and familiar facts concerning the representation theory of compact groups, their Clebsch-Gordan series and coefficients in a general case involving multiplicity, and the definition and properties of unit tensors. All these are used in the main body of the paper.

We shall deal with a general compact semisimple Lie group $G$ of dimension $n$ [except that $\mathrm{U}$ (1) factors will be allowed], and a generic compact Lie subgroup $H$ of dimension $k<n$. The various inequivalent UIR's of $G$ will be labeled by a symbol $J$ which in general comprises a collection of independent quantum numbers. The space of the $J$ th UIR, and its dimension, will be 
written as $\mathcal{H}^{(J)}$ and $N_{J}$, respectively. Within the UIR we use the label $M$ for a complete set of state labels for an orthonormal basis, denoting again several independent quantum numbers. The matrix elements of the UIR matrices $\mathcal{D}^{(J)}$ are written as $\mathcal{D}_{M M^{\prime}}^{(J)}(g), g \in G$. We have

$$
\begin{gathered}
\mathcal{D}^{(J)}(g)^{\dagger} \quad \mathcal{D}^{(J)}(g)=1 \text { on } \mathcal{H}^{(J)}, \\
\mathcal{D}^{(J)}\left(g_{1}\right) \quad \mathcal{D}^{(J)}\left(g_{2}\right)=\mathcal{D}^{(J)}\left(g_{1} g_{2}\right) .
\end{gathered}
$$

The Peter-Weyl theorem gives us the orthogonality and completeness of these matrix elements taken from all UIR's of $G$. With respect to the translation invariant integration measure $d g$ on $G$, normalized to unit total volume, these statements are expressed by

$$
\begin{gathered}
\int_{G} d g \mathcal{D}_{M^{\prime \prime} M^{\prime \prime \prime}}^{\left(J^{\prime}\right)}(g)^{*} \mathcal{D}_{M M^{\prime}}^{(J)}(g)=\delta_{J^{\prime} J} \delta_{M^{\prime \prime} M} \delta_{M^{\prime \prime \prime} M^{\prime}} / N_{J}, \\
\sum_{J M M^{\prime}} N_{J} \mathcal{D}_{M M^{\prime}}^{(J)}(g) \mathcal{D}_{M M^{\prime}}^{(J)}\left(g^{\prime *}=\delta\left(g^{-1} g^{\prime}\right),\right.
\end{gathered}
$$

where $\delta(g)$ is the invariant Dirac delta function on $G$ with respect to $d g$.

When we consider similarly the complete family of UIR's of the subgroup $H \subset G$, we replace the above symbols with the following:

$$
g \rightarrow h, \quad J \rightarrow j, \quad M \rightarrow m, \quad \mathcal{D}^{(J)} \rightarrow D^{(j)}, \quad \mathcal{H}^{(J)} \rightarrow \mathcal{H}^{(j)}, \quad N_{J} \rightarrow N_{j} .
$$

The relations (A2) corresponding to $H$ hold with a normalized integration measure $d h$, and of course $j, m$ are again in general sets of quantum numbers. In particular one may ask for the UIR's of $G$ in a form, or in a basis, adapted to the reduction with respect to $H$. In that case, for each given UIR $J$ of $G$, one has to ask which UIR's $D^{(j)}$ of $H$ are contained within $\mathcal{D}^{(J)}$, and each one with what multiplicity. Then the state label $M$ within $\mathcal{D}^{(J)}$ becomes a triple $\mu j m: j$ and $m$ are the UIR and internal state labels for $H$, while $\mu$ is an (orthonormal) multiplicity label which distinguishes the several occurrences of $D^{(j)}$ within $\mathcal{D}^{(J)}$. If in a particular case the multiplicity is unity, we just set $\mu=1$. Expressed in such a basis, the representation matrices of $G$ appear as $\mathcal{D}_{\mu j m, \mu^{\prime} j^{\prime} m^{\prime}}^{(J)}(g)$, and when $g \in H$ we have

$$
\mathcal{D}_{\mu j m, \mu^{\prime} j^{\prime} m^{\prime}}^{(J)}(h)=\delta_{\mu^{\prime} \mu} \quad \delta_{j^{\prime} j} D_{m m^{\prime}}^{(j)}(h) .
$$

Incidentally for the trivial or identity representations of $G$ or of $H$ we write $J=0, j=0$ respectively, with $M=m=0$ as well.

Now we set up a notation for Clebsch-Gordan coefficients and unit tensor operators, allowing for the possibility of multiplicity in the Clebsch-Gordan series, and for the coefficients to be complex in general. Considering the direct product $\mathcal{D}^{\left(J_{1}\right)} \times \mathcal{D}^{\left(J_{2}\right)}$ of two UIR's of $G$, let the UIR $\mathcal{D}^{\left(J_{3}\right)}$ be present upon reduction, possibly several times, and introduce an orthonormal label $\Lambda$ to distinguish its several occurrences. Then, if $\Psi_{M_{1}}^{\left(J_{1}\right)}, \Psi_{M_{2}}^{\left(J_{2}\right)}$ are orthonormal bases for $\mathcal{H}^{\left(J_{1}\right)}, \mathcal{H}^{\left(J_{2}\right)}$, respectively, for each $\Lambda$ the product vectors

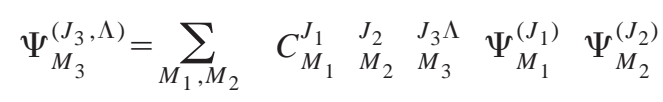

transform by the UIR $J_{3}$ of $G$, and for different $\Lambda$ they are orthogonal. Thus the orthonormality or unitarity and completeness relations for the Clebsch-Gordan coefficients are: 


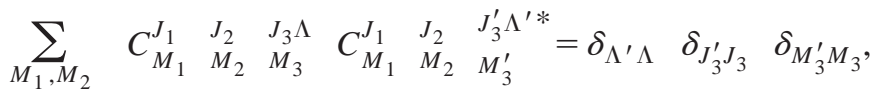

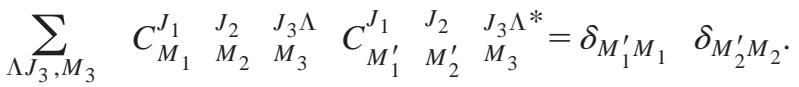

The statement that for each $\Lambda, \Psi_{M_{3}}^{\left(J_{3} \Lambda\right)}$ transforms according to the $\operatorname{UIR} \mathcal{D}^{\left(J_{3}\right)}$ of $G$ leads to

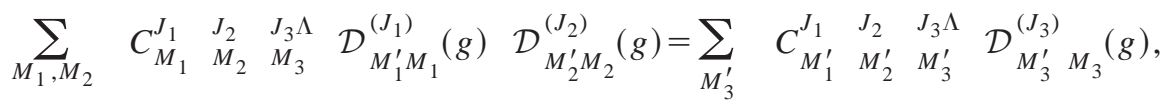

from which follows, using (A5), the result for the product of any two $\mathcal{D}$ matrices:

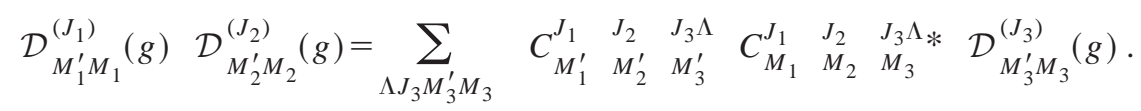

Lastly we consider the Wigner-Eckart theorem, and the definition and properties of unit tensor operators within a UIR. A tensor operator of type $J_{2}$ connecting the two UIR's $J_{1}$ and $J_{3}$ is a collection of operators

$$
T_{M_{2}}^{J_{2}}: \mathcal{H}^{\left(J_{1}\right)} \rightarrow \mathcal{H}^{\left(J_{3}\right)}
$$

obeying the transformation rule

$$
\mathcal{D}^{\left(J_{3}\right)}(g) \quad T_{M_{2}}^{J_{2}} \mathcal{D}^{\left(J_{1}\right)}(g)^{-1}=\sum_{M_{2}^{\prime}} \mathcal{D}_{M_{2}^{\prime} M_{2}}^{\left(J_{2}\right)}(g) T_{M_{2}^{\prime}}^{J_{2}}
$$

The matrix elements of such a set of operators between the two sets of basis states involve a collection of reduced matrix elements labeled by the Clebsch-Gordan multiplicity label $\Lambda$ and accompanied by corresponding Clebsch-Gordan coefficients:

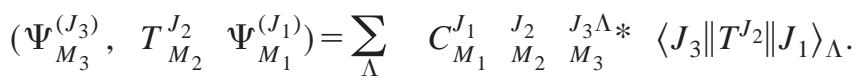

The occurrence of the complex conjugate of the Clebsch-Gordan coefficients is to be noted. One can then express $T_{M_{2}}^{J_{2}}$ explicitly as

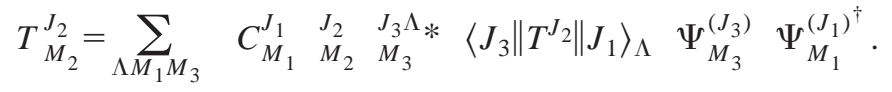

Within a given UIR $\mathcal{D}^{\left(J_{0}\right)}$ of $G$ on $\mathcal{H}^{\left(J_{0}\right)}$, Eq. (A11) leads to the definition of a complete set of unit tensor operators $U_{M}^{J \Lambda}$ as follows:

$$
U_{M}^{J \Lambda}=\sum_{M_{0} M_{0}^{\prime}} C_{M_{0}}^{J_{0}} \begin{array}{lll}
J & J_{0} \Lambda^{*} & M_{0}^{\prime}
\end{array} \Psi_{M_{0}^{\prime}}^{\left(J_{0}\right)} \Psi_{M_{0}}^{\left(J_{0}\right) \dagger}
$$

where we have chosen specially simple values for the reduced matrix elements. These unit tensors obey, as a particular case of (A9):

$$
\mathcal{D}^{\left(J_{0}\right)}(g) \quad U_{M}^{J \Lambda} \quad \mathcal{D}^{\left(J_{0}\right)}(g)^{-1}=\sum_{M^{\prime}} \mathcal{D}_{M^{\prime} M}^{(J)}(g) \quad U_{M^{\prime}}^{J \Lambda}
$$


One can also easily establish their trace orthogonality:

$$
\operatorname{Tr}\left(U_{M^{\prime}}^{J^{\prime} \Lambda^{\prime \dagger}} \quad U_{M}^{J \Lambda}\right)=\frac{N_{J_{0}}}{N_{J}} \delta_{\Lambda^{\prime} \Lambda} \quad \delta_{J^{\prime} J} \quad \delta_{M^{\prime} M}
$$

Therefore any operator $A$ on $\mathcal{H}^{\left(J_{0}\right)}$ is uniquely expressible in the form

$$
\begin{gathered}
A=\sum_{\Lambda J M} a_{M}^{J \Lambda} U_{M}^{J \Lambda}, \\
a_{M}^{J \Lambda}=\frac{N_{J}}{N_{J_{0}}} \operatorname{Tr}\left(U_{M}^{J \Lambda \dagger} A\right) .
\end{gathered}
$$

In Sec. III we have used such formulas in a basis adapted to $H$.

\section{APPENDIX B: INDUCED REPRESENTATIONS ON COSET SPACES AND RECIPROCITY THEOREM}

Here we outline the construction of induced UR's of $G$ starting from UIR's of $H$, and the reciprocity theorem which tells us in detail the irreducible contents of such UR's of $G$. A direct construction of a class of UR's of a semidirect product of $G$ by a certain Ábelian group (similar to the Euclidean and Poincaré groups) proves practically useful in this context.

\section{The inducing construction}

The UIR $D^{(j)}(h)$ of $H$ is defined on the Hilbert space $\mathcal{H}^{(j)}$ of dimension $N_{j}$. Consider functions $\phi: G \rightarrow \mathcal{H}^{(j)}$ satisfying the following (right) covariance law under $H$ :

$$
\begin{gathered}
g \in G \rightarrow \phi(g) \in \mathcal{H}^{(j)}, \\
\phi(g h)=D^{(j)}\left(h^{-1}\right) \quad \phi(g), \\
\phi_{m}(g h)=\sum_{m^{\prime}} D_{m^{\prime} m}^{(j)}(h)^{*} \phi_{m^{\prime}}(g) .
\end{gathered}
$$

(We avoid using letters $\psi, \Psi$ for these vector valued functions on $G$ since they have been used in the main text with specific meanings.) We now define an (left) action by $G$ on such $\phi$ :

$$
(\mathcal{U}(g) \phi)\left(g^{\prime}\right)=\phi\left(g^{-1} g^{\prime}\right) .
$$

The representation property is obvious, and so also the compatibility of the condition (B1) and the action (B2), i.e., the latter respects the former. Let $\Sigma=G / H$ be (as in the text) the space of right cosets in $G$ with respect to $H$, and let $\ell(q)$ be a choice of (local) coset representatives $\Sigma \rightarrow G$. Then it is clear that the independent information in a $\phi$ obeying (B1) is contained in its values at coset representatives:

$$
q \in \Sigma: \quad \phi_{0}(q)=\phi(\ell(q)) .
$$

On these the action by $G$ is easily computed

$$
\mathcal{U}(g) \phi=\phi^{\prime}:
$$




$$
\begin{gathered}
\phi_{0}^{\prime}(q)=\phi^{\prime}(\ell(q))=\phi\left(g^{-1} \ell(q)\right)=\phi\left(\ell\left(g^{-1} q\right) \quad \ell\left(g^{-1} q\right)^{-1} g^{-1} \ell(q)\right) \\
=D^{(j)}\left(\ell(q)^{-1} g \quad \ell\left(g^{-1} q\right)\right)
\end{gathered}
$$

We can now formally define the Hilbert space for these wave functions, in such a way that the operators $\mathcal{U}(g)$ are unitary. We use the following notation:

$$
L^{2}\left(\Sigma, \mathcal{H}^{(j)}\right)=\left\{\phi_{0}(q) \in \mathcal{H}^{(j)} \mid q \in \Sigma,\left\|\phi_{0}\right\|^{2}=\int_{\Sigma} d \mu(q)\left(\phi_{0}(q), \phi_{0}(q)\right)_{\mathcal{H}^{(j)}}<\infty\right\} .
$$

Here $d \mu(q)$ is the $G$-invariant normalized volume element on $\Sigma$, and it is obvious that unitarity of $D^{(j)}$ leads to unitarity of $\mathcal{U}(g)$. This UR of $G$ is said to be induced from the UIR $D^{(j)}$ of $H$, and we will denote it as $\mathcal{D}^{\text {(ind, } j \text { ) }}$ (the dependence on $H$ being left implicit). Combining Eqs. (B4) and (B5) we see that we can introduce an (ideal) orthonormal basis $|q, m\rangle$ for $L^{2}\left(\Sigma, \mathcal{H}^{(j)}\right)$ with these properties:

$$
\begin{gathered}
\phi_{0, m}(q)=\left\langle q, m \mid \phi_{0}\right\rangle, \\
\left\langle q^{\prime}, m^{\prime} \mid q, m\right\rangle=\delta\left(q^{\prime}, q\right) \delta_{m^{\prime} m}, \\
\mathcal{U}(g)|q, m\rangle=\sum_{m^{\prime}} D_{m^{\prime} m}^{(j)}\left(\ell(g q)^{-1} g \ell(q)\right)\left|g q, m^{\prime}\right\rangle .
\end{gathered}
$$

This can be viewed as a standard Wigner form for the UR $\mathcal{D}^{(\text {ind, } j)}$ of $G$.

Now the main question is: how often does the UIR $\mathcal{D}^{(J)}$ of $G$ occur in the UR $\mathcal{D}^{(\text {ind }, j)}$ of $G$, and in case there is nontrivial multiplicity is there a natural way to choose a multiplicity label in an orthonormal manner? To answer these, we turn to a convenient construction of a Master UR of a certain semidirect product group $\mathcal{G}$ involving $G$, originally studied in the context of strong coupling theory. ${ }^{21,22}$

\section{The group $\mathcal{G}$ and the $C G S$ construction}

Choose some UIR $\mathcal{D}^{\left(J_{0}\right)}$ of $G$ (obeying a condition to be given later) and consider a group $\mathcal{G}$ defined as the semidirect product of $G$ by an Ábelian part $P^{\left(J_{0}\right)}$ whose generators belong to $\mathcal{D}^{\left(J_{0}\right)}$. It is convenient to express the structure of $\mathcal{G}$ partly in finite form (the $G$ part) and partly in terms of infinitesimal generators (the Ábelian part). Thus we look for unitary operators $\bar{U}(g), g \in G$, and additional (possibly non-Hermitian) operators $P_{M_{0}}^{\left(J_{0}\right)}$ obeying the relations

$$
\begin{gathered}
\bar{U}\left(g^{\prime}\right) \bar{U}(g)=\bar{U}\left(g^{\prime} g\right), \\
\bar{U}(g) P_{M_{0}}^{\left(J_{0}\right)} \bar{U}(g)^{-1}=\sum_{M_{0}^{\prime}} \mathcal{D}_{M_{0}^{\prime} M_{0}}^{\left(J_{0}\right)}(g) P_{M_{0}^{\prime}}^{\left(J_{0}\right)}, \\
{\left[P_{M_{0}}^{\left(J_{0}\right)}, P_{M_{0}^{\prime}}^{\left(J_{0}\right)} \text { or } P_{M_{0}^{\prime}}^{\left(J_{0}\right)^{\dagger}}\right]=0 .}
\end{gathered}
$$

These relations define $\mathcal{G}$, and the analogy to the structures of $E(3)$ or the Poincare group is evident; therefore we can refer to the $P_{M_{0}}^{\left(J_{0}\right)}$ as momenta. 
We now set up a solution to these relations on the space $\mathcal{H}^{(\mathrm{reg})}=L^{2}(G, \mathcal{C})$ of the regular representation $\mathcal{D}^{\text {(reg) }}$ of $G$. We introduce ideal basis vectors $|g\rangle$ obeying

$$
\left\langle g^{\prime} \mid g\right\rangle=\delta\left(g^{-1} g^{\prime}\right) .
$$

Choose now some numerical (possibly complex) values $p_{M_{0}}^{\left(J_{0}\right)}$ as possible eigenvalues of the $P_{M_{0}}^{\left(J_{0}\right)}$, and define $\bar{U}(g), P_{M_{0}}^{\left(J_{0}\right)}$ on the basis kets $|g\rangle \in \mathcal{H}^{(\mathrm{reg})}$ by

$$
\begin{gathered}
\bar{U}(g)\left|g^{\prime}\right\rangle=\left|g g^{\prime}\right\rangle, \\
P_{M_{0}}^{\left(J_{0}\right)}\left|g^{\prime}\right\rangle=\left(\mathcal{D}^{\left(J_{0}\right)}\left(g^{\prime}\right)^{*} p^{\left(J_{0}\right)}\right)_{M_{0}}\left|g^{\prime}\right\rangle .
\end{gathered}
$$

One can verify that $\bar{U}(g)$ are unitary, and that all the relations (B7) are obeyed, so we have here a certain master UR of $\mathcal{G}$ uniquely specified by the choice of $p^{\left(J_{0}\right)}$. The basis $|g\rangle$ is one in which the momenta are all simultaneously diagonal, and this is the essence of the $C G S$ construction.

This UR of $\mathcal{G}$ can be analyzed in two interesting ways by using two separate bases for $\mathcal{H}^{\text {(reg) }}$. On the one hand, we can exploit the orthogonality and completeness of the UIR's of $G$ as expressed by Eq. (A2), and so introduce a basis $|J M N\rangle$ defined and behaving as follows:

$$
\begin{gathered}
|J M N\rangle=N_{J}^{1 / 2} \int_{G} d g \mathcal{D}_{M N}^{(J)}(g)^{*}|g\rangle, \\
\left\langle J^{\prime} M^{\prime} N^{\prime} \mid J M N\right\rangle=\delta_{J^{\prime} J} \delta_{M^{\prime} M} \delta_{N^{\prime} N}, \\
\bar{U}(g)|J M N\rangle=\sum_{M^{\prime}} \mathcal{D}_{M^{\prime} M}^{(J)}(g)\left|J M^{\prime} N\right\rangle .
\end{gathered}
$$

In this basis in which the regular representation of $G$ is fully reduced, we can exploit the information given in Appendix A to show that the matrix elements of the momenta $P_{M_{0}}^{\left(J_{0}\right)}$ have the following form:

$$
\left\langle J^{\prime} M^{\prime} N^{\prime}\left|P_{M_{0}}^{\left(J_{0}\right)}\right| J M N\right\rangle=\sqrt{\frac{N_{J}}{N_{J^{\prime}}}} \sum_{\Lambda N_{0}} p_{N_{0}}^{\left(J_{0}\right)} C_{M}^{J} \begin{array}{lllllll}
J_{0} & J^{\prime} \Lambda * & M_{0}^{\prime} & M_{N}^{J} & J_{0} & J^{\prime} \Lambda \\
N_{0} & N^{\prime}
\end{array} .
$$

This means that the reduced matrix element of $P^{\left(J_{0}\right)}$ with multiplicity label $\Lambda$ is [see Eq. (A10)].

$$
\left\langle J^{\prime} N^{\prime}\left\|P^{\left(J_{0}\right)}\right\| J N\right\rangle_{\Lambda}=\sqrt{\frac{N_{J}}{N_{J^{\prime}}}} \sum_{N_{0}} p_{N_{0}}^{\left(J_{0}\right)} C_{N}^{J} \begin{array}{llll}
J_{0} & J_{0} & N^{\prime} \Lambda
\end{array} .
$$

We will use this in a moment.

The other way to exploit the CGS construction (B9) is to pass to a description in terms of a coset space. At this point we assume that the stability group of the numerical momentum $p_{M_{0}}^{\left(J_{0}\right)}$ is the subgroup $H \subset G$ :

$$
h \in H: \quad \mathcal{D}^{\left(J_{0}\right)}(h)^{*} \quad p^{\left(J_{0}\right)}=p^{\left(J_{0}\right)} .
$$

Thus the condition on the choice of the UIR $J_{0}$ of $G$ while constructing $\mathcal{G}$ is that $\mathcal{H}^{\left(J_{0}\right)}$ must contain (at least) one $H$-scalar state. We then express a general $g^{\prime} \in G$ as the product $g^{\prime}$ $=\ell(q) h$ of a coset representative and a subgroup element: 


$$
\begin{gathered}
\left.\left|g^{\prime}\right\rangle=\mid q, h\right), \\
\left(q^{\prime}, h^{\prime} \mid q, h\right)=\delta\left(q^{\prime}, q\right) \quad \delta\left(h^{-1} h^{\prime}\right) .
\end{gathered}
$$

Then Eq. (B9) appears as

$$
\begin{gathered}
\left.\bar{U}(g) \mid q, h)=|g \ell(q) h\rangle=\left|\ell(g q) \ell(g q)^{-1} g \ell(q) h\right\rangle=\mid g q, \ell(g q)^{-1} g \ell(q) h\right), \\
\left.\left.P_{M_{0}}^{\left(J_{0}\right)} \mid q, h\right)=\left(\mathcal{D}^{\left(J_{0}\right)}(\ell(q))^{*} p^{\left(J_{0}\right)}\right)_{M_{0}} \mid q, h\right) .
\end{gathered}
$$

The key point is that in the last relation the eigenvalues of the momenta are independent of $h$, precisely because of Eq. (B13). To arrive at basic states behaving in the Wigner form (B6) under $\bar{U}(g)$ we just have to exploit the regular representation of $H$ in the same way as we $\operatorname{did}$ for $G$ in Eq. (B10). So from $\mid q, h)$ we pass to $|q, j m n\rangle$,

$$
\begin{gathered}
\left.|q, j m n\rangle=N_{j}^{1 / 2} \int_{H} d h D_{m n}^{(j)}(h)^{*} \mid q, h\right), \\
\left\langle q^{\prime}, j^{\prime} m^{\prime} n^{\prime} \mid q, j m n\right\rangle=\delta\left(q^{\prime}, q\right) \delta_{j^{\prime} j} \delta_{m^{\prime} m} \delta_{n^{\prime} n} .
\end{gathered}
$$

In this basis we find

$$
\begin{gathered}
\bar{U}(g)|q, j m n\rangle=\sum_{m^{\prime}} D_{m^{\prime} m}^{(j)}\left(\ell(g q)^{-1} g \ell(q)\right)\left|g q, j m^{\prime} n\right\rangle, \\
P_{M_{0}}^{\left(J_{0}\right)}|q, j m n\rangle=\left(\mathcal{D}^{\left(j_{0}\right)}(\ell(q))^{*} p^{\left(J_{0}\right)}\right)_{M_{0}}|q, j m n\rangle .
\end{gathered}
$$

All the operators of $\mathcal{G}$, both $\bar{U}(g)$ and $P_{M_{0}}^{\left(J_{0}\right)}$, conserve the quantum numbers $j$ and $n$. So if these are kept fixed, and only $q$ and $m$ are allowed to vary, we see that we have exactly recovered Eq. (B6). This shows that the $C G S$ UR of $\mathcal{G}$ corresponding to a $p_{M_{0}}^{\left(J_{0}\right)}$ with stability group $H \subset G$ contains each induced UR $\mathcal{D}^{(\text {ind, } j)}$ of $G$ exactly $N_{j}$ times.

On the other hand, we can link up now to the results (B10) in the basis $|J M N\rangle$ by adapting the choice of labels $M, N, \ldots$ to reduction with respect to the subgroup $H$. As described in Appendix A, this makes $M, N \ldots$ into triples $\mu k m, \nu j n, \ldots$, and then Eq. (B10) and (B12) become

$$
\begin{aligned}
& \bar{U}(g)|J \quad \mu k m \quad \nu j n\rangle=\sum_{\mu^{\prime} k^{\prime} m^{\prime}} \mathcal{D}_{\mu^{\prime} k^{\prime} m^{\prime}, \mu k m}^{(J)}(g)\left|J \quad \mu^{\prime} k^{\prime} m^{\prime} \quad \nu j n\right\rangle,
\end{aligned}
$$

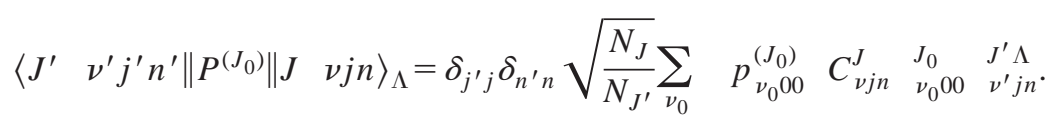

There are as many independent components to $p^{\left(J_{0}\right)}$ as there are $H$-scalar states in $\mathcal{D}^{\left(J_{0}\right)}$. So while $\bar{U}(g)$ conserve $\nu j n, P_{M_{0}}^{\left(J_{0}\right)}$ conserve only $j$ and $n$, but not the multiplicity labels $\nu^{\prime}$ and $\nu$. Realizing that from the original basis $|g\rangle$ for $\mathcal{H}^{(\mathrm{reg})}$ we have arrived in two ways, via the sequences $|g\rangle \rightarrow|J M N\rangle \rightarrow|J \quad \mu k m \quad \nu j n\rangle$ and $|g\rangle \rightarrow \mid q, h) \rightarrow|q, j m n\rangle$, at two alternative bases for the same UR of $\mathcal{G}$, in which the actions by $\bar{U}(g)$ and $P_{M_{0}}^{\left(J_{0}\right)}$ are, respectively, given by Eq. (B18) and Eq. (B17), we come to the following conclusions:

$$
S p(|J \mu k m \quad \nu j n\rangle \mid J \mu k m \nu \text { varying, } j n \text { fixed })=S p(|q, j m n\rangle \mid q, m \text { varying, } j n \text { fixed }),
$$


and the corresponding subspace of $\mathcal{H}^{(\text {reg) }}$ carries exactly once the induced UR $\mathcal{D}^{(\text {ind }, j)}$ of $G$. Comparing this with the reduced matrix element result (B18) we then see that this UR of $G$ contains the UIR $\mathcal{D}^{(J)}$ of $G$ as often as $\mathcal{D}^{(J)}$ contains the UIR $D^{(j)}$ of $H$, which is the reciprocity theorem; the index $\nu$ catalog (in an orthonormal way) these several occurrences of $\mathcal{D}^{(J)}$.

We appreciate that in the final statement of the reciprocity theorem the representation $\mathcal{D}^{\left(J_{0}\right)}$ and the momenta $P_{M_{0}}^{\left(J_{0}\right)}$ have disappeared; they play only an intermediate role in the CGS construction and in recognizing that we have two equally good bases for the Hilbert space carrying the UR $\mathcal{D}^{(\text {ind, } j)}$ of $G$.

${ }^{1}$ E. P. Wigner, Phys. Rev. 40, 749 (1932).

${ }^{2}$ M. Hillery, R. F. O'Connell, M. O. Scully, and E. P. Wigner, Phys. Rep. 106, 121 (1984); Y. S. Kim and M. E. Noz, Phase Space Pictures of Quantum Mechanics (World Scientific, Singapore, 1991).

${ }^{3} \mathrm{H}$. Weyl, The Theory of Groups and Quantum Mechanics (Dover, New York, 1931), p. 274.

${ }^{4}$ P. A. M. Dirac, Rev. Mod. Phys. 17, 195 (1945).

${ }^{5}$ H. J. Groenewold, Physica (Utrecht) 12, 405 (1946); J. E. Moyal, Proc. Cambridge Philos. Soc. 45, 99 (1949).

${ }^{6}$ K. E. Cahill and R. J. Glauber, Phys. Rev. 177, 1857 (1969); 177, 1882 (1969); G. S. Agarwal and E. Wolf, Phys. Rev. D 2, 2161 (1970); 2, 2187 (1970); 2, 2206 (1970).

${ }^{7}$ R. J. Glauber, Phys. Rev. 131, 2766 (1963); E. C. G. Sudarshan, Phys. Rev. Lett. 10, 277 (1963).

${ }^{8}$ K. Husimi, Proc. Phys. Math. Soc. Jpn. 22, 264 (1940); J. Kano, J. Math. Phys. 6, 1913 (1965); C. L. Mehta and E. C. G. Sudarshan, Phys. Rev. 138, B274 (1965).

${ }^{9}$ J. R. Klauder, J. Math. Phys. 4, 1058 (1963).

${ }^{10}$ A. M. Perelomov, Commun. Math. Phys. 26, 222 (1972).

${ }^{11}$ J. M. Radcliffe, J. Phys. A 4, 343 (1971); F. T. Arecchi, E. Courtens, R. Gilmore, and H. Thomas, Phys. Rev. A 6, 2211 (1972).

${ }^{12}$ G. S. Agarwal, Phys. Rev. A 24, 2889 (1981).

${ }^{13}$ J. R. Klauder and E. C. G. Sudarshan, Fundamentals of Quantum Optics (W. A. Benjamin, New York, 1968); L. Mandel and E. Wolf, Rev. Mod. Phys. 37, 231 (1965).

${ }^{14}$ A. P. Balachandran et al., "A fuzzy lens space $L(3)$ and its *-product," Syracuse University preprint.

${ }^{15}$ J. R. Klauder and B. Skagerstam, Coherent States: Applications in Physics and Mathematical Physics (World Scientific, Singapore, 1985), pp. 23 and 34.

${ }^{16}$ C. Brif and A. Mann, J. Phys. A 31, L9 (1998); Phys. Rev. A 59, 971 (1999). See also, J. M. Garcia-Bondiá and J. C. Várilly, J. Phys. A 18, L879 (1988); J. C. Várilly and J. M. Garcia-Bondiá, Ann. Phys. (N.Y.) 190, 107 (1989): J. F. Cariñena, J. M. Garcia-Bondiá, and J. C. Várilly, J. Phys. A 23, 901 (1990).

${ }^{17}$ G. W. Mackey, Group Representations in Hilbert Space (AMS, Providence, RI, 1963).

${ }^{18}$ L. K. Pandit and N. Mukunda, J. Math. Phys. 6, 1547 (1965).

${ }^{19}$ F. Haake and M. Wilkens, "Quasi probabilities based on squeezed states" in Photons and Quantum Fluctuations, Malvern Physics Series, edited by E. R. Pike and H. Walther (Adam Hilger, New York, 1988); J. Stat. Phys. 53, 345 (1988).

${ }^{20}$ S. M. Roy and V. Singh, Phys. Rev. D 25, 3413 (1982); M. Ventata Satyanarayana, ibid. 32, 400 (1985); F. A. M. de Oliviera, M. S. Kim, P. L. Knight, and V. Buzek, Phys. Rev. A 41, 2645 (1990); P. Kral, J. Mod. Opt. 37, 889 (1990).

${ }^{21}$ C. Goebel, Phys. Rev. Lett. 16, 1130 (1966); T. Cook and B. Sakita, J. Math. Phys. 8, 708 (1967).

${ }^{22}$ N. Mukunda, J. Math. Phys. 10, 97 (1969). 